ARTICLE

\title{
Distinct mechanisms of the human mitoribosome recycling and antibiotic resistance
}

\author{
Ravi Kiran Koripella (10 1, Ayush Deep ${ }^{1}$, Ekansh K. Agrawal', Pooja Keshavan (1) ${ }^{1}$, Nilesh K. Banavali (i) ${ }^{1,2}$ \& \\ Rajendra K. Agrawal (iD 1,2四
}

Ribosomes are recycled for a new round of translation initiation by dissociation of ribosomal subunits, messenger RNA and transfer RNA from their translational post-termination complex. Here we present cryo-EM structures of the human $55 \mathrm{~S}$ mitochondrial ribosome (mitoribosome) and the mitoribosomal large 395 subunit in complex with mitoribosome recycling factor $\left(R R F_{m t}\right)$ and a recycling-specific homolog of elongation factor $G\left(E F-G 2_{m t}\right)$. These structures clarify an unusual role of a mitochondria-specific segment of RRF $\mathrm{mt}_{\text {, }}$ identify the structural distinctions that confer functional specificity to $E F-G 2_{m t}$, and show that the deacylated tRNA remains with the dissociated 39S subunit, suggesting a distinct sequence of events in mitoribosome recycling. Furthermore, biochemical and structural analyses reveal that the molecular mechanism of antibiotic fusidic acid resistance for $\mathrm{EF}-\mathrm{G} 2_{\mathrm{mt}}$ is markedly different from that of mitochondrial elongation factor $\mathrm{EF}-\mathrm{G} 1_{\mathrm{mt}}$, suggesting that the two human $E F-G_{m t} s$ have evolved diversely to negate the effect of a bacterial antibiotic.

\footnotetext{
${ }^{1}$ Division of Translational Medicine, Wadsworth Center, New York State Department of Health, Empire State Plaza, Albany, NY, USA. ${ }^{2}$ Department of

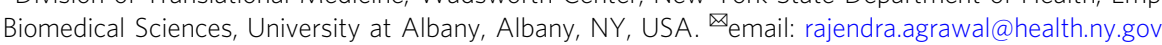


T he process of protein synthesis in all living cells is orchestrated by highly complex macromolecular assemblies called ribosomes, in coordination with mRNA, tRNAs, and multiple translational factors. Mitochondrial ribosomes (mitoribosomes) and their associated translation machinery are distinct from those in the cytoplasm and display features reminiscent of prokaryotic translation ${ }^{1}$, in line with the assumption that mitochondria have evolved from endocytosis of an $\alpha$-proteobacterium by an ancestral eukaryotic cell ${ }^{2}$. However, cryo-electron microscopy (cryo-EM) structures have revealed that the mammalian mitoribosomes have diverged considerably from bacterial ribosomes and acquired several unique features ${ }^{3-8}$. A striking difference is the reversal in the protein to RNA ratio, as the bacterial ribosomes are high in ribosomal RNA (rRNA) whereas the mammalian mitoribosomes are high in protein. The increase in protein mass is the result of acquisition of multiple mito-specific ribosomal proteins (MRPs) and addition of extensions to many MRPs, that are homologous to bacterial ribosomal proteins. Though the steps of mitochondrial translation closely resemble those for prokaryotic translation in the general sequence of events and the homologous accessory protein factors involved, they also show significant structural and functional differences ${ }^{9,10}$.

The complex process of protein synthesis is accomplished in four essential steps of initiation, elongation, termination, and the ribosome recycling. The transition between translation termination and ribosome recycling is well characterized in eubacteria. During translation termination, the nascent polypeptide chain attached to the peptidyl tRNA is released from the ribosome with the help of a class I release factor (RF) that interacts with the stop codon exposed at the ribosomal decoding site, or aminoacyltRNA binding site (A site) ${ }^{11,12}$. Subsequently, the class I RF is dissociated from the ribosome with the help of a class II RF in a GTP hydrolysis-dependent manner ${ }^{13,14}$. At the end of the termination, the translated mRNA and the deacylated tRNA remain associated with the ribosome ${ }^{15,16}$, a state referred to as the posttermination complex (PoTC). In order to initiate a new round of protein synthesis, the ribosome must be split into its two subunits and its bound ligands must be removed. In eubacteria, the disassembly of the PoTC requires the concerted action of two protein factors, the ribosome recycling factor (RRF) and the elongation factor $\mathrm{G}(\mathrm{EF}-\mathrm{G})^{15,17,18}$. RRF binds to the PoTC as the 70 S ribosome adopts a ratcheted conformation ${ }^{19,20}$, in which the small (30S) subunit of the ribosome rotates in an anticlockwise direction with respect to the large $(50 \mathrm{~S})$ subunit $^{21}$. This is followed by the binding of EF-G in conjugation with guanosine $5^{\prime}$ triphosphate (GTP) to the RRF-bound PoTC and the dissociation of the $70 \mathrm{~S}$ ribosome into its large and small subunits, a process that requires the hydrolysis of GTP on EF-G ${ }^{22-24}$. Though the involvement of a third factor, initiation factor 3 (IF3) in the recycling process is generally agreed upon, its precise function has been debated ${ }^{16,18,23}$.

Unlike most eubacteria, where a single form of EF-G is known to participate in both the elongation and ribosome recycling steps $^{22,25}$, mammalian mitochondria utilize two isoforms of EF$\mathrm{G}, \mathrm{EF}-\mathrm{G} 1_{\mathrm{mt}}$, and EF-G2 $2_{\mathrm{mt}}{ }^{26,27}$. While $\mathrm{EF}-\mathrm{G} 1_{\mathrm{mt}}$ specifically functions as a translocase during the polypeptide elongation step ${ }^{28}$, EF-G2 $2_{\mathrm{mt}}$ has been reported to act exclusively as a second recycling factor together with $\mathrm{RRF}_{\mathrm{mt}}{ }^{27}$. Human $\mathrm{RRF}_{\mathrm{mt}}$ is about $25-30 \%$ identical to its eubacterial homologs but carries an additional 79 amino acids (aa) long extension at its $\mathrm{N}$-terminus ${ }^{29}$. The recent high-resolution cryo-EM structures of $\mathrm{RRF}_{\mathrm{mt}}$ bound to the $55 \mathrm{~S}$ mitoribosomes ${ }^{7}$, and an in vivo formed mitoribosomal complex ${ }^{30}$ revealed that the structurally conserved segment of the $\mathrm{RRF}_{\mathrm{mt}}$ is similar to its bacterial analog on $14,19,20,31-36$ and off 37,38 the $70 \mathrm{~S}$ ribosome in terms of its overall size and domain composition. However, the unique mito-specific $\mathrm{N}$-terminal extension
(NTE) in $\mathrm{RRF}_{\mathrm{mt}}$ extends towards the GTPase-associated center and interacts with the functionally important $16 \mathrm{~S}$ rRNA elements of the mitoribosomal $39 \mathrm{~S}$ subunit, including the rRNA helices 89 (H89), H90, and $\mathrm{H}_{92}{ }^{7}$.

Valuable mechanistic inferences about the bacterial ribosome recycling process were made from the structures of the 70S.RRF (e.g., Agrawal et al. ${ }^{31}$ ) and dissociated 50S•RRF•EF-G ${ }^{14,20} \mathrm{com}$ plexes. Capturing the simultaneous binding of both factors on the $70 \mathrm{~S}$ ribosome is challenging, however, owing to the rapid rate of $70 \mathrm{~S}$ ribosomes dissociation into subunits by the combined action of RRF and EF-G ${ }^{22}$. To slow down this reaction, a heterologous system with T. thermophilus RRF and E. coli EF-G was used to capture both factors on the $70 \mathrm{~S}$ ribosome by cryo-EM ${ }^{34}$. Subsequently, a time-resolved cryo-EM study was also able to capture various 70 S.RRF.EF-G functional intermediates, albeit at low resolution ${ }^{36}$. More recently, a bacterial ribosome recycling complex containing both RRF and EF-G was obtained by X-ray crystallography by stabilizing EF-G on the 70 S ribosome through a fusion between EF-G and ribosomal protein bL9 ${ }^{35}$. All these structures conclude that binding of EF-G to the 70S.RRF complex induces rotation of RRF domain II towards the helix 44 (h44) region of the $30 \mathrm{~S}$ subunit, destabilizing the crucial intersubunit bridges $\mathrm{B} 2 \mathrm{a}$ and $\mathrm{B} 3$, and thereby facilitating the dissociation of the $70 \mathrm{~S}$ ribosome into its two subunits.

With a molecular weight of $87 \mathrm{kD}$, human $\mathrm{EF}-\mathrm{G} 2_{\mathrm{mt}}$ is slightly larger than $E F-G 1_{m t}(83 \mathrm{kD})$, as well as the two isoforms of bacterial EF-Gs, EF-G (78 kD), and EF-G2 (73 kD), identified in certain bacterial species but without known function for the second bacterial isoform ${ }^{39-41}$, except in case of a spirochete ${ }^{42}$. EF-G2 $2_{m t}$ has about $36 \%$ aa sequence identity to $E F-G 1_{m t}$ and about $30 \%$ aa identity to both its bacterial homologs. Some mammalian mitochondrial translation steps are now better understood through determination of the cryo-EM structures of the initiation ${ }^{43-45}$ and the elongation ${ }^{6,46}$ complexes at highresolution. Our previous study of the human mitoribosome recycling complex of the $R F_{m t}$-bound $55 S^{7}$ provided useful insights into the mito-specific aspects of the recycling process, but a complete 55S mitoribosomal recycling complex comprising both $\mathrm{RRF}_{\mathrm{mt}}$ and $\mathrm{EF}-\mathrm{G}_{\mathrm{mt}}$ remained elusive.

In this work, we investigate the role of mito-specific aa segments of $\mathrm{RRF}_{\mathrm{mt}}$ and EF-G2 $2_{\mathrm{mt}}$ in recycling the $55 \mathrm{~S}$ mitoribosome PoTC by determining cryo-EM structures of the key intermediate mitoribosome $\cdot \mathrm{RRF}_{\mathrm{m}} \cdot \mathrm{EF}-\mathrm{G} 2_{\mathrm{mt}}$ complexes, and determine the effect of fusidic acid, an antibiotic that is known to inhibit the GTPase activity of bacterial EF-G ${ }^{47}$, on the GTPase activity of EF$\mathrm{G} 2{ }_{\mathrm{mt}}$. Our study reveals distinct features of the mechanism of human mitoribosome recycling and of the mechanism of fusidicacid resistance shown by $\mathrm{EF}-\mathrm{G} 1_{\mathrm{mt}}$ and $\mathrm{EF}-\mathrm{G} 2_{\mathrm{mt}}$.

\section{Results and discussion}

Structure of the human mitoribosome recycling complex. To investigate the molecular mechanism of ribosome recycling in mammalian mitochondria, we first prepared a model posttermination complex (PoTC) by incubating the human 55S mitoribosome with puromycin ${ }^{7,48}$. The model PoTC was briefly incubated with human $\mathrm{RRF}_{\mathrm{mt}}$ and human $\mathrm{EF}-\mathrm{G} 2_{\mathrm{mt}}-\mathrm{GMPPCP}$ to obtain the mitoribosome recycling complex. (see "Methods" section). Single-particle cryo-EM analysis on this complex yielded three major classes that each represent a major functional state formed during human mitoribosome recycling, referred henceforth to as Class I, Class II, and Class III (Fig. S1). Class I corresponds to the intact $55 \mathrm{~S}$ mitoribosome that carries a strong density for $R F_{m t}$, and was refined to $3.5 \AA$ (Figs. 1 and S1a). Class II, a relatively small class with only 28,929 particle images, corresponds to the $55 \mathrm{~S}$ mitoribosome that carries both $\mathrm{RRF}_{\mathrm{mt}}$ and 

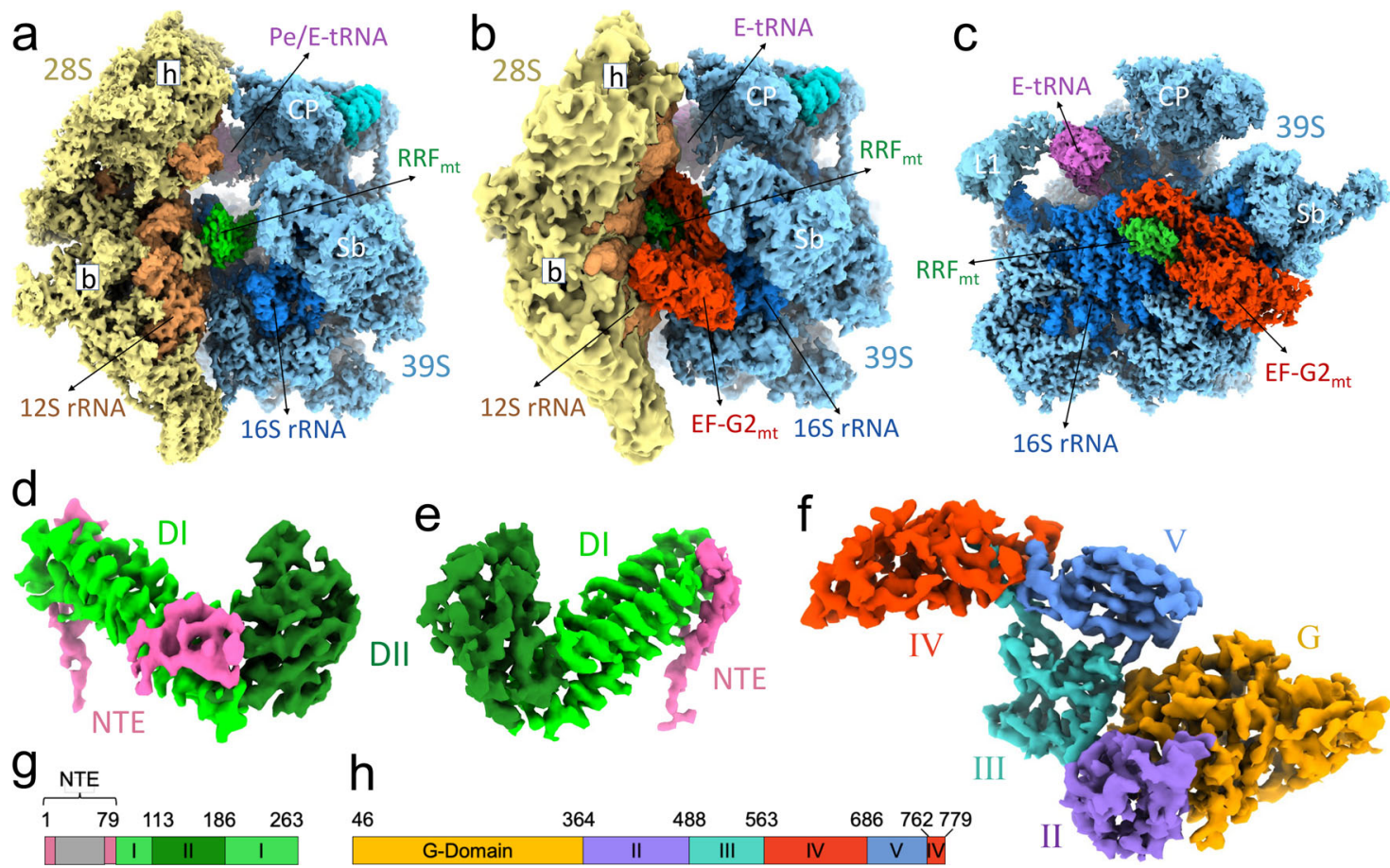

h
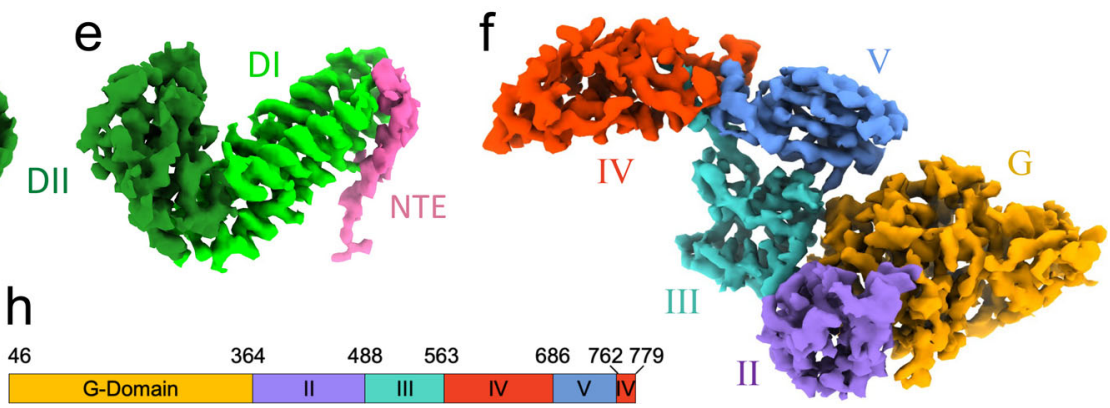

Fig. 1 Cryo-EM structures of the human mitochondrial recycling complexes in three functional states. Segmented cryo-EM maps of the mitoribosomal

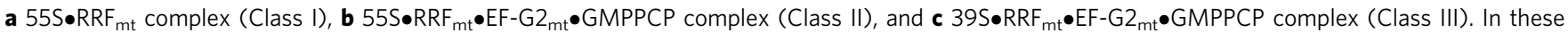
three panels, the $28 \mathrm{~S}$ subunit is shown in yellow, the $39 \mathrm{~S}$ subunit in blue, E-tRNA in orchid, RRF $\mathrm{mt}_{\mathrm{t}}$ in green and EF-G2 $2_{\mathrm{mt}}$ in red. A lighter shade of yellow differentiates the $28 \mathrm{~S}$ ribosomal proteins from the 12S rRNA, while a lighter shade of blue differentiates the $39 \mathrm{~S}$ ribosomal proteins from the $16 \mathrm{~S}$ rRNA. Landmarks of the $28 \mathrm{~S}$ subunit: h, head; b, body. Landmarks of the $39 \mathrm{~S}$ subunit: CP central protuberance, Sb stalk base; L1, MRP uL1m. d Cryo-EM density of $\mathrm{RRF}_{\mathrm{mt}}$ extracted from the Class I complex. e Cryo-EM density of RRF $\mathrm{mt}_{\text {t }}$ extracted from the Class III complex. $\mathbf{f}$ Cryo-EM density of EF-G2 ${ }_{\text {mt }}$ extracted from Class III complex. See Figs. S1 and S2, for overall and local resolutions, respectively, of densities corresponding to the mitoribosome, RRF ${ }_{\mathrm{mt}}$ and $\mathrm{EF}-\mathrm{G} 2_{\mathrm{mt}}$ in these complexes. $\mathbf{g}$ Domain organization in $\mathrm{RRF}_{\mathrm{mt}}$ displaying domain I (light green), domain II (dark green), modeled region of NTE (pink) and unmodelled region of NTE (gray) h Domain organization in EF-G2 ${ }_{m t}$ showing G domain (orange), domain II (purple), domain III (cyan), domain IV (red) and domain V (blue).

EF-G2 ${ }_{\mathrm{mt}}$, and was refined to $3.9 \AA$ (Fig. S2b). In this class, the densities corresponding to the large (39S) mitoribosomal subunit, $\mathrm{RRF}_{\mathrm{mt}}$, and EF-G2 $2_{\mathrm{mt}}$ are well resolved, but the small (28S) mitoribosomal subunit appears to be loosely bound and present in multiple poses (Figs. 1 and S2). The most populated Class III corresponds to the dissociated $39 \mathrm{~S}$ subunits that carry both $\mathrm{RRF}_{\mathrm{mt}}$ and EF-G2 $2_{\mathrm{mt}}$, and was refined to $3.15 \AA$ (Figs. 1 and S2c). Class II likely represents an ensemble of low-population intermediate states of mitoribosome recycling that occur between the states represented by Classes I and III. In addition to these three recycling complexes, we have also obtained a class of particles consisting of $55 \mathrm{~S}$ mitoribosomes without either of the two factors, where the $28 \mathrm{~S}$ subunit was rotated by about $8^{\circ}$ around its long axis such that its shoulder side moves closer to the $39 \mathrm{~S}$ subunit while its platform side moves away from it (Fig. S3a). A similar orientation for the small subunit relative to the large subunit, termed as "subunit rolling" has been reported earlier for the $80 \mathrm{~S}$ ribosomes $^{49}$ and the $55 \mathrm{~S}$ mitoribosomes ${ }^{3,7}$.

$\mathrm{RRF}_{\mathrm{mt}}$ binding stabilizes the rotated state of the $28 \mathrm{~S}$ subunit in the 55S mitoribosome. Superimposition of our Class I complex with the $\mathrm{RRF}_{\mathrm{mt}}$-unbound human ${ }^{3}$, bovine ${ }^{8}$ and porcine ${ }^{4} 55 \mathrm{~S}$ mitoribosomes showed that the small $28 \mathrm{~S}$ subunit was rotated counter-clockwise by about $8.5^{\circ}$ with respect to the large $39 \mathrm{~S}$ subunit (Fig. S3b), similar to the "ratchet-like inter-subunit rotation" observed in the bacterial $70 \mathrm{~S}$ ribosome $\mathrm{e}^{21}$ and the $55 \mathrm{~S}$ mitoribosomes complexed with translational factors $6,7,44,46$. In addition to the inter-subunit rotation, the head domain of the $28 \mathrm{~S}$ subunit is rotated by about $4^{\circ}$ towards the tRNA exit (E) site in a direction roughly orthogonal to the inter-subunit motion (Fig. S3b), similar to "head swiveling" in the bacterial $70 \mathrm{~S}$ ribosomes ${ }^{50,51}$. As expected, the structure of the Class I complex matches the previously published $3.9 \AA$ resolution map of the analogous $55 \mathrm{~S} \cdot \mathrm{RRF}_{\mathrm{mt}}$ complex ${ }^{7}$.

The Class I map showed the characteristic "L" shaped $R R_{\mathrm{mt}}$ density and a density corresponding to a pe/E-state tRNA within the inter-mitoribosomal subunit space. The overall positioning and domain arrangement of $\mathrm{RRF}_{\mathrm{mt}}$ in the Class I map is similar to the bacterial RRF on ${ }^{14,19,20,31-36}$ and off ${ }^{37}$ the $70 \mathrm{~S}$ ribosomes, and also to the structures of RRF bound to the $70 \mathrm{~S}$ chloroplast ribosome $\mathrm{e}^{52,53}$, and $\mathrm{RRF}_{\mathrm{mt}}$ bound to the human mitochondrial $55 \mathrm{~S}$ in our previous study ${ }^{7}$. As observed in bacteria, domain I is positioned close to the peptidyl transferase center (PTC) and extends towards the $\alpha$-sarcin-ricin loop (SRL). A striking difference between the human $\mathrm{RRF}_{\mathrm{mt}}$ and its bacterial counterpart is the presence of a 79 aa long N-terminal extension (NTE) in $\mathrm{RRF}_{\mathrm{mt}}$. We could model the last 14 aa residues of the NTE of $\mathrm{RRF}_{\mathrm{mt}}$ into an additional density contiguous with the a-helix1 from domain I. As discussed in our previous study ${ }^{7}$, the NTE is strategically positioned in the intersubunit space between domain I and several functionally important 16S rRNA structural elements such as H89, H90, H92 (A-loop), and MRP L16 (Fig. S4) and interacts with several nucleotides (nts) and aa residues in its vicinity ${ }^{7}$. Interestingly, unlike the $\alpha$-helical nature inferred for the part of this segment of $\mathrm{NTE}^{7}$, we find that its higher resolution density to be partially unstructured. A similar observation of a relatively unstructured NTE has been recently 

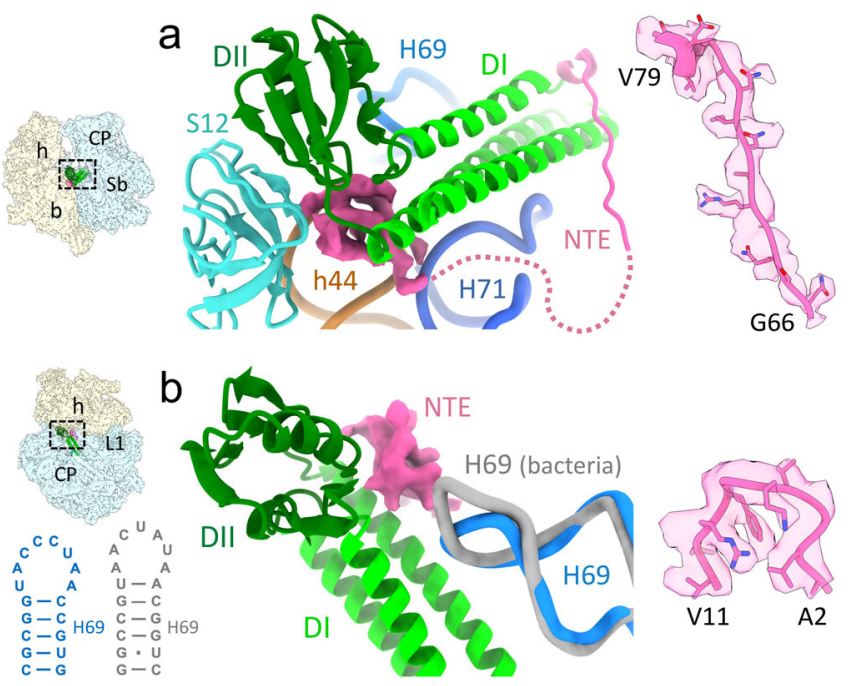

\section{MALGLKCFRMVHPTFRNYLAASIRPVSEVTLKTVHERQHG} HRQYMAYSAVPVRHFATKKAKAKGKGOSQTRVNINAALV

Fig. 2 Structures of two NTE segments of $\mathbf{R R F}_{\mathbf{m t}}$. a Density corresponding to the $\mathrm{N}$-terminus segment of RRF $\mathrm{mt}_{\mathrm{m}} \mathrm{NTE}$ (pink) observed in the intersubunit space sandwiched between the $28 \mathrm{~S}$ SSU components, including MRP uS12m (cyan) and the 12S rRNA helix h44 (brown), the 39S LSU components, including $16 \mathrm{~S}$ rRNA helices $\mathrm{H} 69$ (medium blue) and $\mathrm{H} 71$ (dark blue), and the outer bent of the junction between domains I and II of RRF $\mathrm{mt}_{\text {. }}$. The dotted line (pink) depicts the connection between two structurally stabilized segments of the RRF ${ }_{m t}$ 's NTE. b H69 superimposition from bacterial (gray) 33 and human mitochondrial ribosomes (blue) reveals the shortening of $\mathrm{H} 69$ in the mitoribosome, also depicted in secondary structures of $\mathrm{H} 69$ on the lower left. Domain II of RRF $m$ interacts with the shorter $\mathrm{H} 69$ through its strategically positioned N-terminus of NTE (pink). c Sequence of NTE showing two modeled regions (pink). Thumbnails to the left depicts an overall orientation of the 555 mitoribosome, with semitransparent 285 (yellow) and 395 (blue) subunits, and overlaid positions of ligands. Landmarks on the thumbnail: $h$, head, and $b$, body of the $28 \mathrm{~S}$ subunit, and CP central protuberance, Sb stalk base of the $39 \mathrm{~S}$ subunit.

reported in an in vivo state complex ${ }^{30}$. However, the mitoribosomal components interacting with $\mathrm{RRF}_{\mathrm{mt}}{ }^{7}$ essentially remain unaltered.

We found a small density in a tight pocket surrounded by the outer bend of the junction between domains I and II of $\mathrm{RRF}_{\mathrm{mt}}$, MRP uS12m and the small subunit's $12 \mathrm{~S}$ rRNA helix h44, and the large subunit's 16S rRNA helices H69 and H71 (Fig. 2a). Except for our previous lower resolution map of the $55 \mathrm{~S} \cdot \mathrm{RRF}_{\mathrm{mt}}$ complex ${ }^{7}$, this additional density is not observed in any of the available $55 \mathrm{~S}$ mitoribosomal structures, whether complexed with other translational factors $6,44,46$ or not ${ }^{3,4}$. Since our complex was reconstituted from purified components, this additional density should correspond to an $\mathrm{RRF}_{\mathrm{mt}}$ NTE segment, that has been stabilized through interactions with multiple mitoribosomal components in its vicinity. Though bacterial and mitochondrial ribosomes exhibit significant differences in their overall shape, composition, and conformation, their internal rRNA core regions are largely conserved ${ }^{3-5,8}$. Comparison of the RRF binding sites between the bacterial and mitochondrial ribosomes reveals that the H69 of $16 \mathrm{~S}$ rRNA is slightly shorter in the mammalian mitoribosomes (Fig. 2b). This minor shortening of H69 is critical because it directly impacts the interaction of $\mathrm{H} 69$ with domain II of $\mathrm{RRF}_{\mathrm{mt}}$. The small density, most likely corresponding to $\mathrm{N}$ terminus segment of the mito-specific NTE, appears to compensate for the shortened $\mathrm{H} 69$ by mediating the interactions between $\mathrm{RRF}_{\mathrm{mt}}$ and $\mathrm{H} 69$ (Fig. 2b). We generated a model that would place 10 aa residues (Ala2-Val11) at the N-terminus of NTE into this density (Fig. 2b). This model was guided by the observed sidechain density for two consecutive large side chains of Phe8 and Arg9 within the first 11 aa (Fig. 2c). Since there are other possibilities for two consecutive aa residues with large sidechains in the remaining 54 aa residues in the unmodelled segment of NTE, further confirmation of this assignment may need a well-resolved density for the entire NTE.

It should be noted that the $\mathrm{RRF}_{\mathrm{mt}}$-bound $55 \mathrm{~S}$ mitoribosomes (present work and ref. ${ }^{7}$ ) were never observed in the unrotated state, suggesting that the $\mathrm{RRF}_{\mathrm{mt}}$ binding locks the ribosome in a fully rotated state. This is in contrast to the bacterial 70S•RRF complexes that were found both in their rotated and unrotated conformational states $14,19,20,31-36$. The rotated conformation of the $55 \mathrm{~S}$ mitoribosome seems to prime subunit dissociation by either destabilizing or completely breaking seven out of fifteen inter-subunit bridges in the unrotated $55 \mathrm{~S}$ mitoribosome ${ }^{3,8}$. The simultaneous interactions of $\mathrm{N}$-terminus segment of the $\mathrm{RRF}_{\mathrm{mt}}$ NTE with $R_{R F}$ 's structurally conserved domain I, MRP uS12m, h44, H69, and H71 likely help prevent the back-rotation of the small $28 \mathrm{~S}$ subunit. The rotated state of the $55 \mathrm{~S}$ mitoribosome could serve as an ideal substrate for the subsequent binding of EF$\mathrm{G} 2 \mathrm{mt}$ to complete subunit dissociation. In this context, it is important to note that the entire 79 aa long NTE is an integral part of the mature protein and is known to be essential for $\mathrm{RRF}_{\mathrm{mt}}$ function during the mitoribosome recycling 29,54 .

$\mathrm{RRF}_{\mathrm{mt}}$ domain II motion helps split the $55 \mathrm{~S}$ mitoribosome into its two subunits. Using fast-kinetics, it has been shown that the splitting/recycling of the 70S ribosome by the concerted action of RRF and EF-G happens in the sub-second time scale ${ }^{22}$. Both RRF and EF-G have been captured on the 70S ribosome with timeresolved cryo-EM ${ }^{36}$. It is more challenging without time-resolved techniques, but RRF and EF-G were also captured on the $70 \mathrm{~S}$ ribosomes by using the factors from different species $^{34}$ or by crosslinking the EF-G with one of the ribosomal proteins ${ }^{35}$. In the present work, collection of very large cryo-EM datasets (altogether 21,752 micrographs (Fig. S1), enabled the isolation of a small subset of $55 \mathrm{~S}$ particles (Class II) that contained both $\mathrm{RRF}_{\mathrm{mt}}$ and EF-G2 $\mathrm{mt}$ (Fig. 1). However, the $28 \mathrm{~S}$ subunit density was found to be weak and present in multiple destabilized conformations relative to the $39 \mathrm{~S}$ subunit in this complex.

Both Class II and Class III maps showed readily recognizable densities corresponding to $\mathrm{RRF}_{\mathrm{mt}}, \mathrm{EF}-\mathrm{G} 2_{\mathrm{mt}}$., and E-site tRNA. The Class III complex had superior resolution, which enabled more accurate analysis of molecular-level interactions between the two factors and the mitoribosome, and their functional implications, while the Class II map was useful for interpreting large-scale conformational changes. In line with the bacterial 70S/ $50 \mathrm{~S} \bullet \mathrm{RRF} \bullet \mathrm{EFG}$ complexes ${ }^{14,20,34-36}$, the conformation of $\mathrm{RRF}_{\mathrm{mt}}$ is substantially different between the Class I and Class III recycling complexes. The conformation of $\mathrm{RRF}_{\mathrm{mt}}$ domain I remains unchanged among all three classes. In the Class II and III maps, domain II was rotated by about $45^{\circ}$ towards the small subunit compared to its position in the Class I complex (Fig. 3a). This large conformational change is enabled by a highly flexible hinge regions between domain $I$ and domain II in $R_{R F}$. Due to this rotation, the tip of domain II moved by about $40 \AA$ towards the h44 of $12 \mathrm{~S}$ rRNA. When the maps of Class II and III were superimposed, this motion resulted in a major steric clash between the $\mathrm{RRF}_{\mathrm{mt}}$ domain II and the 28S subunit elements h44 and MRP uS12m (Fig. 3a). In the 55S mitoribosome, h44 is involved in the formation of two intersubunit bridges B2a and B3 by pairing with $\mathrm{H} 69$ and $\mathrm{H} 71$, respectively ${ }^{3,8}$. By displacing h44, 

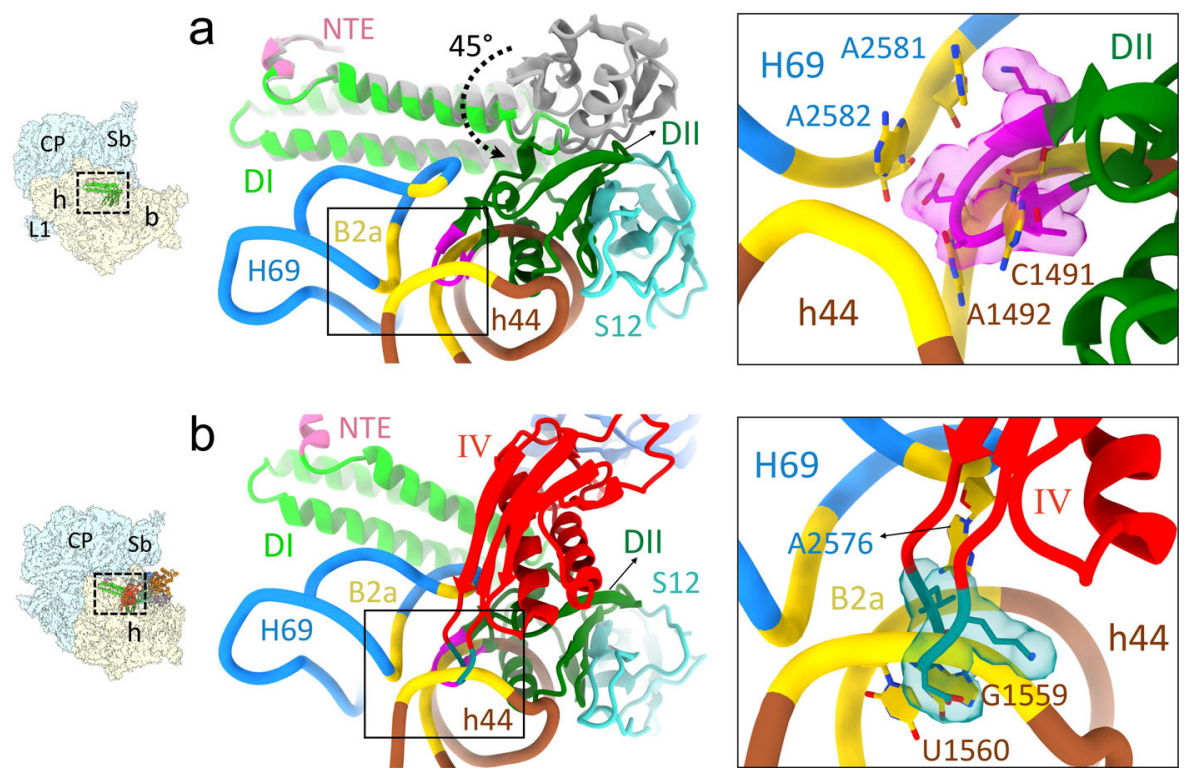

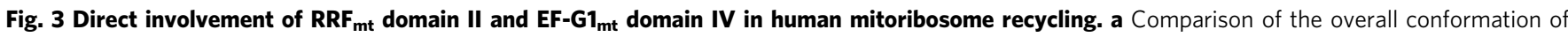
$\mathrm{RRF}_{\mathrm{mt}}$ between the Class I (gray) and Class III (green) complexes revealed that domain II rotates by about $45^{\circ}$ towards the $28 \mathrm{~S}$ subunit. Such a rotation would sterically clash with the $12 \mathrm{~S}$ rRNA helix 44 (h44, brown) and MRP uS12m (cyan) and destabilize a crucial inter-subunit bridge, B2a (yellow). Inset shows the magnified view of the inter-subunit bridge B2a with RRF $_{\mathrm{mt}}$ domain II residues V121-K127 (shown with density in magenta) disrupting B2a by inserting between h44 residues (C1491 and A1492) and H69 residues (A2581 and A2582). b Superimposition of the Class III complex with the Class I complex shows a direct overlap between the loop1 region (dark cyan) of EF-G2 ${ }_{\mathrm{mt}}$ domain IV (red) and the intersubunit bridge B2a (yellow) formed between h44 (brown) and H69 (blue). Inset shows the magnified view of EF-G2 $\mathrm{mt}$ domain IV residues L582-R585 (shown with density in dark cyan) that will disrupt the bridge B2a by inserting between h44 residues (G1559 and U1560) and H69 residue (A2576) and pushing the 28S subunit (h44) away. Thumbnails to the left depict an overall orientation of the $55 \mathrm{~S}$ mitoribosome, with semitransparent $28 \mathrm{~S}$ (yellow) and 39S (blue) subunits, and overlaid position RRF $\mathrm{mt}_{\text {. }}$ Landmarks on the thumbnails are same as in Fig. 2.

$\mathrm{RRF}_{\mathrm{mt}}$ disrupts these crucial intersubunit bridges, thereby splitting the $55 \mathrm{~S}$ mitoribosome into its two subunits.

A well-defined $28 \mathrm{~S}$ structure was not seen within the Class II $55 \mathrm{~S}$ mitoribosomal complex, but several inter-subunit bridges do appear to be destabilized or broken, and the small subunit seems to be dissociating from the large subunit. To remove any possible large subunit contamination from the Class II map, extensive reference-based $3 \mathrm{D}$ classification was employed, but this class of 55 S mitoribosomal particles with a well-resolved 39S subunit and a poorly resolved $28 \mathrm{~S}$ subunit remained unchanged. This supports it being an ensemble of authentic, short-lived functional intermediates of mitoribosome recycling, where the small subunit is captured in multiple positions during its separation from the large subunit.

EF-G2 $2_{m t}$ binding induces conformational changes in $R_{R F}$. The $55 \mathrm{~S} \cdot \mathrm{RRF}_{\mathrm{mt}}$ complex (Class I) undergoes large conformational changes upon binding to EF-G2 ${ }_{\mathrm{mt}}$ (Classes II and III). EF$\mathrm{G} 2{ }_{\mathrm{mt}}$ would not be able to access its binding site on the mitoribosome without the significant movement seen in domain II of $\mathrm{RRF}_{\mathrm{mt}}$ in Class II and Class III complexes. This movement eliminates the direct spatial conflicts of EF-G2 ${ }_{\mathrm{mt}}$ domains III, IV, and $\mathrm{V}$ with the initial position of $\mathrm{RRF}_{\mathrm{mt}}$ domain II in the Class I complex (Figs. 3a, b and Fig. S5). This change could be induced either during or upon binding of EF-G2 ${ }_{\mathrm{mt}}$. The domain II of $\mathrm{RRF}_{\mathrm{mt}}$ is repositioned into a cavity created by domains III, IV, and V of EF-G2 $\mathrm{mt}$ (Fig. S6a) and an extensive network of interactions are formed between the two mitochondrial recycling factors. This is also in agreement with the location of RRF domain II reported in the bacterial $50 \mathrm{~S}^{14}$ and $70 \mathrm{~S}$ complexes ${ }^{34,36}$. A majority of the interactions are formed between the hinge regions that connect the two domains of $\mathrm{RRF}_{\mathrm{mt}}$ and the loop regions from domain III of EF-G2 $2_{\mathrm{mt}}$. Several aa residues from the $\mathrm{RRF}_{\mathrm{mt}}$ hinge region (Pro183-Thr186) interact with EF-G2 $2_{\mathrm{mt}}$ domain III residues Glu495-Leu499 (Fig. S6b) via hydrogen bonds and hydrophobic interactions. Arg187 from the $\alpha$-helix following the hinge region of $\mathrm{RRF}_{\mathrm{mt}}$ domain II has close hydrogen-bonding interactions with Tyr556 of EF-G2 ${ }_{\mathrm{mt}}$ domain III (Fig. S6b). A second set of contacts formed between the two factors involves residues Ile109-Arg110 from the second hinge region of $\mathrm{RRF}_{\mathrm{mt}}$ and residues Ser527-Gln529 from the domain III of EF-G2 (Fig. S6c).

Domain IV of EF-G2 $2_{\mathrm{mt}}$ presses against domain II of $\mathrm{RRF}_{\mathrm{mt}}$ through multiple interactions. The surface residues of $a$-helix 1 (Asn629, Ser633, and Leu636) and a-helix 2 (Thr664, Met665, Ser667, and Ala668) from domain IV of EF-G2 $2_{\mathrm{mt}}$ interact with residues in $\beta$-strand 3 (Ser134-Met138), and its adjoining loop region (Gln132 and Ile133) from domain II of $\mathrm{RRF}_{\mathrm{mt}}$ through a combination of electrostatic and hydrophobic interactions (Fig. S6d). Gln637 from the a-helix 1 of EF-G2 ${ }_{\mathrm{mt}}$ also shares a hydrogen bond with Ser112 from the hinge region of $R_{R F}$ (Fig. S6d). Contacts are observed between the C-terminal $\alpha$-helix of EF-G2 $2_{\mathrm{mt}}$ domain IV and the a-helix 3 from the triple-helix bundle of $\mathrm{RRF}_{\mathrm{mt}}$ domain I. In bacteria, the analogous C-terminal a-helix of EF-G is often considered as part of domain $\mathrm{V}$ though the first atomic models of EF-G ${ }^{55,56}$ grouped it with domain IV. While residues Ser776-Leu778 from EF-G2 ${ }_{\mathrm{mt}}$ domain IV pair with residues Arg251 and Val255 from $\mathrm{RRF}_{\mathrm{mt}}$ domain I through hydrogen bonds (Fig. S6e), Arg775 from EF-G2 ${ }_{m t}$ domain IV strongly interacts with Glu259 from $\mathrm{RRF}_{\mathrm{mt}}$ domain I through a salt-bridge (Fig. S6e). Direct interactions of EF-G2 ${ }_{\mathrm{mt}}$ domain III with $\mathrm{RRF}_{\mathrm{mt}}$ domain II at its hinge regions, known to confer interdomain flexibility to the bacterial factor ${ }^{38}$, likely help trigger the dissociation of $55 \mathrm{~S}$ mitoribosomes into subunits by enabling the repositioning of $\mathrm{RRF}_{\mathrm{mt}}$ domain II. At the same time, the 

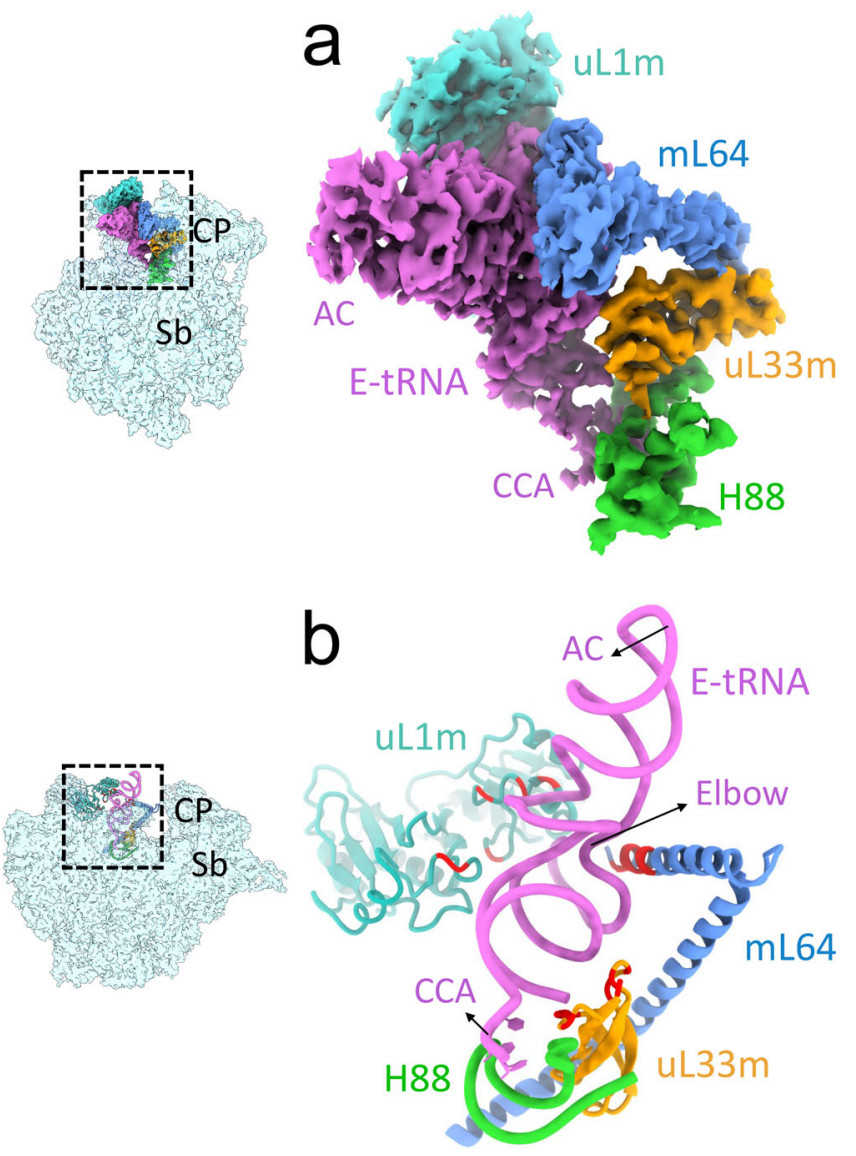

Fig. 4 Stabilization of the E-site tRNA on the dissociated 39S subunit. a Extracted cryo-EM densities corresponding to E-site tRNA and interacting 395 subunit components. The E-site tRNA (orchid) is stabilized on the 395 subunit through multiple interactions with the mitoribosomal components. The elbow region of the E-site tRNA is sandwiched between 39S MRPs uL1m (cyan) and the mitospecific $\mathrm{mL} 64$ (blue) while the acceptor arm and the CCA end have strong interactions with uL33m and the 16S rRNA helix H88, respectively. b Atomic models for the densities shown in a. Multiple MRP segments bearing positively charged aa residues (Arg and Lys; red) interact with the negatively charged phosphates of the E-site tRNA molecule. To better display the location of the positively charged aa residues at the interface of tRNA and MRPs, the $\mathbf{b}$ orientation is obtained by applying an upward rotation around a horizontal axis to the panel a orientation. AC and CCA refer to anticodon and CCA ends of the TRNA. Thumbnails to the left depict an overall orientation of the 395 subunit (semitransparent blue), with overlaid highlighted mitoribosomal components and ligands. Landmarks on the thumbnail: CP central protuberance, Sb stalk base.

multiple interactions between domain IV of EF-G $2_{\mathrm{mt}}$ and domain II of $\mathrm{RRF}_{\mathrm{mt}}$ appear to stabilize the $\mathrm{RRF}_{\mathrm{mt}}$ domain II in the altered position, which would push the $28 \mathrm{~S}$ subunit away from the $39 \mathrm{~S}$ subunit and prevent domain II from reverting back to its previous orientation, in order to maintain the $39 \mathrm{~S} \bullet \mathrm{RRF}_{\mathrm{mt}} \bullet \mathrm{EF}-\mathrm{G} 2_{\mathrm{mt}}$ complex in a dissociated state.

In addition to aiding to the function of $R_{R F}, E F-G 2_{m t}$ plays a direct role in destabilizing the $55 \mathrm{~S}$ mitoribosome. Superimposition of the maps of Class I and Class III complexes reveals a direct steric clash between the loop1 region of EF-G2 $2_{\mathrm{mt}}$ domain IV and the $28 \mathrm{~S}$ subunit component (rRNA h44), that participates in the formation of the inter-subunit bridge B2a (Fig. 3b). More importantly, the orientation of domain IV loopl seems to be unique to $\mathrm{EF}-\mathrm{G} 2{ }_{\mathrm{mt}}$ since the analogous region in $\mathrm{EF}-\mathrm{G} 1_{\mathrm{mt}}$ is positioned away from the intersubunit bridge $\mathrm{B} 2 \mathrm{a}$ towards the decoding center 6,46 .

Role of mito-specific MRPs in stabilizing the deacylated tRNA. The presence of E-site tRNA in Class III complex of the dissociated 39S subunit suggests a markedly different mechanism of deacylated tRNA removal during recycling of mitochondrial PoTC as compared to that in the bacteria, where the deacylated tRNA goes with the small ribosomal subunit during the $70 \mathrm{~S}$ ribosome splitting ${ }^{36}$. This observation is particularly intriguing since a majority (11 of 12) of the rRNA segments in bacterial $23 \mathrm{~S}$ rRNA that are known to interact with the E-site tRNA are absent in the mito-16S rRNA ${ }^{57}$. We find that the E-site tRNA in the 39S complex is stabilized by multiple interactions with Arg-rich and Lys-rich electro-positive segments of MRPs, including $\mathrm{mL}^{6}{ }^{6}$, $\mathrm{uL} 33 \mathrm{~m}$, and $\mathrm{uL} 1 \mathrm{~m}^{58}$ (Fig. 4). With the tightly held body of the deacylated tRNA through conserved interaction of its CCA end with the rRNA H88 and multiple new interactions with MRPs, transition of its anticodon end from a pe/E state ${ }^{7}$ in Class I complex to E/E-state in Class II and III complexes could in part facilitate the dissociation of the two mitoribosomal subunits. Moreover, the mechanism of subsequent release of deacylated tRNA from the $39 \mathrm{~S}$ subunit remains unknown.

Dynamic interactions among $\mathrm{uL11}$, CTD of uL12m, and EFG2 $2_{\mathrm{mt}}$. EF-G2 $2_{\mathrm{mt}}$ binding resulted in a prominent conformational change in the uL11m stalk-base region of the $39 \mathrm{~S}$ subunit. The $\mathrm{uL} 11 \mathrm{~m}$ stalk-base region moved towards the CTD of $\mathrm{uL} 12 \mathrm{~m}$, a component of the L10-L12 stalk, and assumed a unique conformation not reported previously $3,4,6,7,44$. The $16 \mathrm{~S}$ rRNA H43 of the $\mathrm{uL} 11 \mathrm{~m}$ stalk-base moved by $3 \AA$ towards the CTD of $\mathrm{uL} 12 \mathrm{~m}$, oriented parallel to the domain V of EF-G2 $2_{\mathrm{mt}}$, while the NTD of MRP uL11m moved about $5 \AA$ away from the EF-G2 $2_{\mathrm{mt}}$ domain V (Fig. 5a). This is in sharp contrast to the 55S•EF-G1 $1_{\mathrm{mt}}$ translocation complexes $3,4,6,7,44,46$, where the $\mathrm{uL} 11 \mathrm{~m}$ stalk-base region was observed to move $5 \AA$ closer towards the domain $\mathrm{V}$ of EF$\mathrm{G} 1_{\mathrm{mt}}$ (Fig. 5b). The movement of $\mathrm{uL} 11 \mathrm{~m}$ away from the EF-G2 $2_{\mathrm{mt}}$ domain $\mathrm{V}$ and towards the CTD of $\mathrm{uL} 12 \mathrm{~m}$ is essential for the binding of EF-G2 ${ }_{\mathrm{mt}}$ in the present conformation, to avoid the steric clash between the NTD of $\mathrm{uL} 11 \mathrm{~m}$ and domain V of EF- $\mathrm{G}_{\mathrm{mt}}$ (Fig. 5a). It is also possible that the conformation of EF-G2 $\mathrm{mt}$ observed in our $39 \mathrm{~S} \cdot \mathrm{EF}-\mathrm{G} 2{ }_{\mathrm{mt}}$ complex (Class III) was attained after the dissociation of $39 \mathrm{~S}$ subunit from the $55 \mathrm{~S}$ complex (Class II).

In addition to the unique conformation of the uL11m stalk-base region, the $\mathrm{CTD}$ of $\mathrm{uL} 12 \mathrm{~m}$ was also observed in a distinct conformation. In the $55 \mathrm{~S} \cdot \mathrm{EF}-\mathrm{G} 1_{\mathrm{mt}}$ complexes $^{6,46}$, the CTD of $\mathrm{uL} 12 \mathrm{~m}$ is positioned close to EF-G1 $1_{\mathrm{mt}}$ so that $\alpha$-helices 1 and 2 of $\mathrm{uL} 12 \mathrm{~m}$ CTD would have close interactions with the $\mathrm{G}^{\prime}$ subdomain of EF-G1 $1_{\mathrm{mt}}$ (Fig. 5c), thereby providing stability to the otherwise flexible $\mathrm{uL} 12 \mathrm{~m}$ CTD. In contrast, the CTD of $\mathrm{uL} 12 \mathrm{~m}$ rotates by about $60^{\circ}$ and shifts away by about $7 \AA$ from the $\mathrm{G}^{\prime}$ subdomain of EF-G2 $2_{\mathrm{mt}}$ in the $39 \mathrm{~S} \cdot \mathrm{EF}-\mathrm{G} 2_{\mathrm{mt}}$ complex (Fig. $5 \mathrm{~d}$ ). As a result of this large rotational movement, interactions between the uL12m CTD a-helix 2 and the $\mathrm{G}^{\prime}$ subdomain of EF-G2 $2_{\mathrm{mt}}$ are lost, while the contacts between the $a$-helix 1 and the $\mathrm{G}^{\prime}$ subdomain of EF-G2 $\mathrm{mt}$ are maintained (Fig. $5 \mathrm{~d}$ ). Since protein $\mathrm{uL} 12$ is known to play a central role in the recruitment of translational factors to the bacterial ribosome ${ }^{59-62}$; the semi-stable conformation of $\mathrm{uL} 12 \mathrm{~m}$ observed in the $39 \mathrm{~S} \cdot \mathrm{EF}-\mathrm{G} 2 \mathrm{mt}$ complex represents a late-stage conformation of $\mathrm{uL} 12 \mathrm{~m}$ prior to its detachment from the EF-G2 as $\mathrm{EF}-\mathrm{G} 2{ }_{\mathrm{mt}}$ 's participation in the $55 \mathrm{~S}$ ribosome recycling process nears completion. 

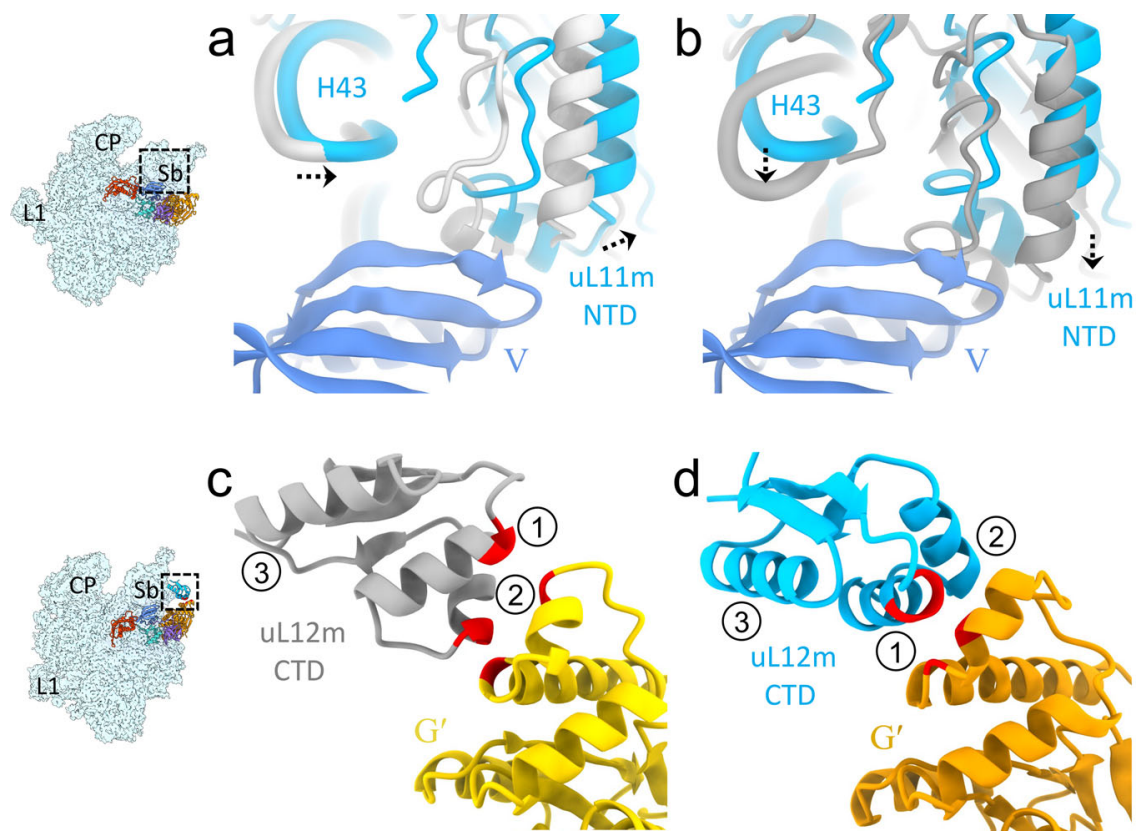

Fig. 5 EF-G2 $2_{m t}$ binding induces large-scale conformational changes in the uL11m stalk-base region and the CTD of uL12m. a In the $39 S \bullet R R F_{m t} \bullet E F-G 2_{m t}$ complex, the uL $11 \mathrm{~m}$ stalk-base region (blue) moves towards the CTD of uL12m and away from the domain $\mathrm{V}$ of EF-G2 $2_{\mathrm{mt}}$, as compared to its position in the $\mathrm{EF}-\mathrm{G} 2_{\mathrm{mt}}$-unbound $55 \mathrm{~S}$ mitoribosome (light gray) ${ }^{3}$. $\mathbf{b}$ In sharp contrast, in the presence of EF-G1 $\mathrm{mt}_{\mathrm{t}}$, the uL $11 \mathrm{~m}$ stalk-base region was found to move towards the domain $\mathrm{V}$ of $\mathrm{EF}-\mathrm{G} 1_{\mathrm{mt}}$ in the translocation complex (dark gray) ${ }^{6}$. c In the $55 \mathrm{~S} \bullet \mathrm{EF}-\mathrm{G} 1_{\mathrm{mt}}$ complex $^{6}$, the CTD of uL12m (gray) is positioned in such a way that its $\alpha$-helices 1 and 2 directly interact with the $\mathrm{G}^{\prime}$ subdomain of EF-G1 $1_{\mathrm{mt}}$ (yellow). $\mathbf{d}$ In our $39 \mathrm{~S} \bullet R R F_{\mathrm{mt}} \bullet \mathrm{EF}-\mathrm{G} 2_{\mathrm{mt}}$ Complex, the CTD of uL12m (blue) is rotated by about $60^{\circ}$ and shifted away by about $7 \AA$ from the $\mathrm{G}^{\prime}$ subdomain of $\mathrm{EF}-\mathrm{G} 2_{\mathrm{mt}}$, resulting in the loss of contacts between its $\alpha$-helix 2 and $\mathrm{G}^{\prime}$ subdomain of EF-G2 $2_{\mathrm{mt}}$ while a new set of interactions is formed between its $\alpha$-helix 1 and $\mathrm{G}^{\prime}$ subdomain of EF-G2 $\mathrm{mt}$. Thumbnails to the left depict an overall orientation of the $39 \mathrm{~S}$ subunit (semitransparent blue), and overlaid positions of the ligands. Landmarks on the thumbnail: CP central protuberance, Sb stalk base, L1 MRP uL1m.

Structural basis for use of EF-G2 $2_{\mathrm{mt}}$ in mitoribosomal recycling instead of EF-G1 $\mathbf{m}_{\mathbf{m t}}$. In most bacterial species, a single EF-G is involved in both translocation and ribosome recycling. Mammalian mitoribosomes have evolved to utilize the two isoforms EF-G1 $1_{m t}$ and EF-G2 $2_{m t}$ to cope with the significantly altered environment in mitochondria as compared to the bacterial cytoplasm $^{26,27}$ and perform two separate functions ${ }^{26,27}$. EF-G1 $\mathrm{mt}$ is used during translation elongation while EF-G $2_{\mathrm{mt}}$ is used along with $\mathrm{RRF}_{\mathrm{mt}}$ for the recycling of the $55 \mathrm{~S}$ mitoribosome. The overall position and domain arrangement of EF-G2 $2_{\mathrm{mt}}$ is similar to that of EF-G $1_{m t}$ in the recently published human ${ }^{6}$ and porcine ${ }^{46}$ translocational complexes (Fig. S6). There are, however, some specific structural distinctions between the two factors that assign them specialized functional roles. The most striking of these is the presence of a C-terminal extension (CTE) in EF-G1 $1_{\mathrm{mt}}$ domain IV (Fig. 6a $)^{6}$. Besides its CTE, the size of the conserved C-terminal $\alpha$ helix of EF-G1 $1_{\mathrm{mt}}$ domain IV is substantially longer (16 aa) (Fig. 6a) than the C-terminal $\alpha$-helix (12 aa) of EF-G2 ${ }_{\mathrm{mt}}$ domain IV (Fig. 6b).

The sturdier and longer C-terminal $\alpha$-helix of EF-G1 $1_{m t}$ domain IV and its 11 aa CTE would not permit the coexistence of $R R F_{m t}$ on the mitoribosome due to a major steric clash between domain I of $\mathrm{RRF}_{\mathrm{mt}}$ and the C-terminal region of EF-G1 $1_{\mathrm{mt}}$ (Fig. $6 \mathrm{~b}$ ). Even a reorientation of the $\mathrm{C}$-terminal $\alpha$-helix and its CTE away from the domain I of $\mathrm{RRF}_{\mathrm{mt}}$ would not resolve the problem as they would then clash with $\mathrm{H} 89$ of the 16S rRNA and the NTE of $\mathrm{RRF}_{\mathrm{mt}}$, that has been positioned in the inter subunit space between the domain I of $\mathrm{RRF}_{\mathrm{mt}}$ and 16S rRNA helix, H89 (Fig. 6b). Structural analysis of the bacterial EF-Gs from various species ${ }^{47,63,64}$ has revealed that the length of their C-terminal $\alpha$ helices are about 12 aa long, suggesting that only EF-G2 $2_{\mathrm{mt}}$ can function alongside $\mathrm{RRF}_{\mathrm{mt}}$ during subunit splitting. Interestingly, the C-terminal a-helices of EF-G2 from T. thermophilus ${ }^{39}$ and $\mathrm{EF}-\mathrm{G} 2_{\mathrm{mt}}$ are similar in size (Fig. S7) though the function of EFG2 in $T$. thermophilus is not understood. Moreover, the interaction between the C-terminal regions of EF-G2 $2_{\mathrm{mt}}$ domain IV and $\mathrm{RRF}_{\mathrm{mt}}$ domain $\mathrm{I}$ is essential for stabilizing the bound $\mathrm{RRF}_{\mathrm{mt}}$. This tight anchoring of $\mathrm{RRF}_{\mathrm{mt}}$ domain $\mathrm{I}$ to the mitoribosome would prevent $\mathrm{RRF}_{\mathrm{mt}}$ dissociation from the mitoribosome, when its domain II undergoes substantial rotation to displace the h44 region of the $28 \mathrm{~S}$ subunit. This agrees with the observation that deletion of the last few aa residues from the Cterminal region of bacterial RRF adversely effects its function during $70 \mathrm{~S}$ ribosome recycling ${ }^{65}$.

Now the question is why EF-G1 $1_{\mathrm{mt}}$ mediates the translocation step during mitoribosomal elongation and not EF-G2 $2_{\mathrm{mt}}$ ? The most probable answer is that the CTE in the domain IV of EF$\mathrm{G} 1_{\mathrm{mt}}$ that limits its ability to participate in the mitochondrial ribosome recycling, is directly involved in the elongation step of mammalian mitochondrial protein synthesis ${ }^{6}$. By contacting the inner bend of the A-site tRNA, the CTE of EF-G1 $1_{m t}$ can help in translocating the CCA arm of the A-site tRNA into the P site, and by interacting with a $16 \mathrm{~S}$ rRNA helix, H71, it prevents the P-site tRNA from reverting back into the A site ${ }^{6}$. This analysis is further supported by the fact that bacterial EF-Gs, which lack the CTE, are inactive on the $55 \mathrm{~S}$ mitochondrial ribosomes while EF-G1 $1_{\mathrm{mt}}$ is active on the $70 \mathrm{~S}$ ribosomes ${ }^{66}$.

Specific sequence differences in a critical region (loop1) within the domain IV of EF-G2 $2_{\mathrm{mt}}$ can also make it ineffective in driving translocation. During EF-G-catalyzed translocation in bacteria, the presence of two universally conserved glycine residues at the tip of domain IV (loop 1) region facilitate the insertion of domain IV into the decoding center (DC) ${ }^{47}$. In the DC, the loop 1 of domain IV destabilizes the codon-anticodon interactions of the 


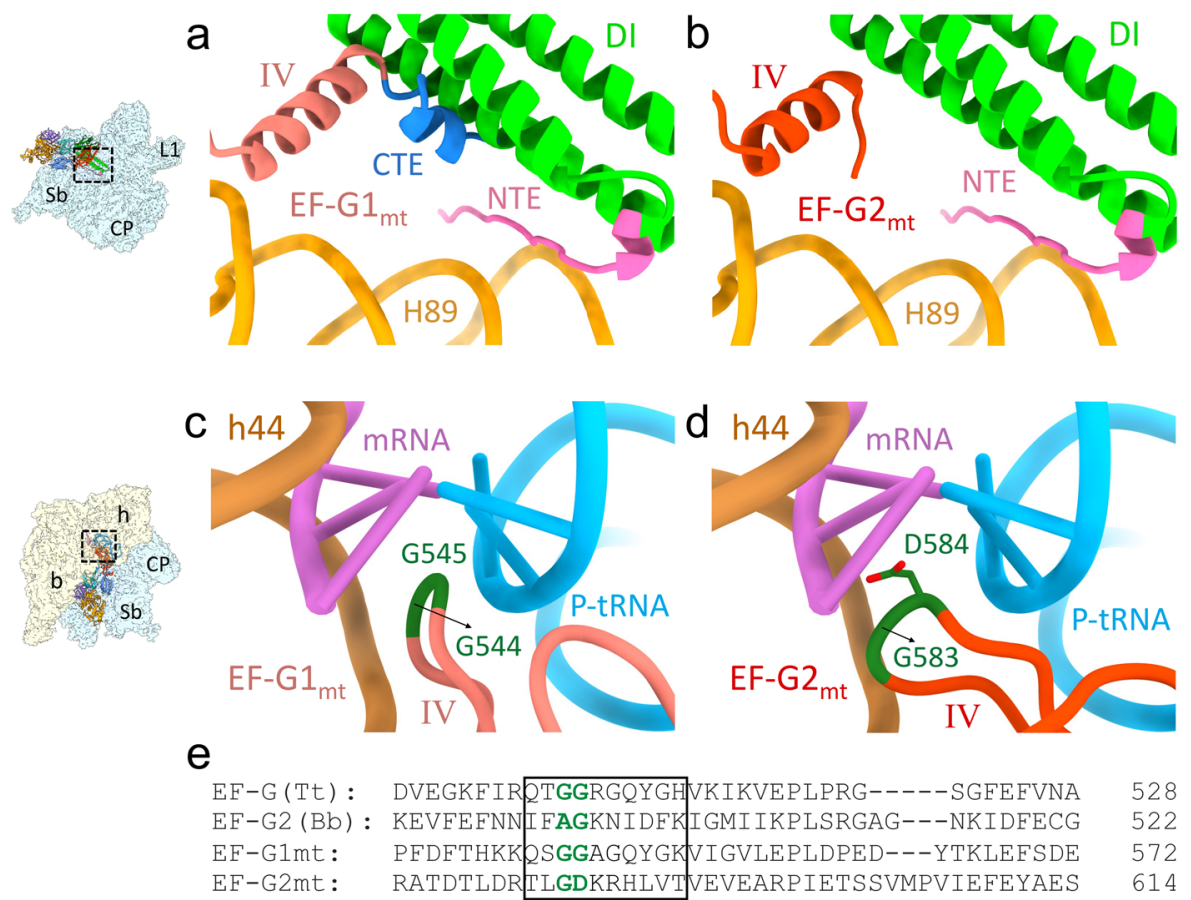

Fig. 6 Structural basis for the exclusive roles of EF-G1 $\mathbf{m}_{\mathbf{m t}}$ in $\mathbf{~ R N A}$ translocation and EF-G2 $\mathbf{m b}_{\mathbf{m}}$ in mitoribosome recycling. a The presence of a substantially longer C-terminal $\alpha$-helix (salmon) along with the presence of a unique CTE (blue), which is required for mitochondria tRNA translocation ${ }^{6}$, prevents the simultaneous binding of $\mathrm{EF}_{-} \mathrm{G1}_{\mathrm{mt}}$ and $\mathrm{RRF}_{\mathrm{mt}}$ (green) on the mitoribosome due to a major steric clash. Furthermore, any conformational repositioning the $\mathrm{C}$ terminal $\alpha$-helix of EF-G1 $1_{m t}$ will result in direct steric clash with the $\mathrm{H} 89$ (orange) of $16 \mathrm{~S}$ rRNA and the NTE of RRF $\mathrm{mt}$ (pink). $\mathbf{b}$ Having a shorter C-terminal $\alpha$-helix (red) and the absence of CTE allows the simultaneous binding of EF-G2 $2_{\mathrm{mt}}$ and $\mathrm{RRF}_{\mathrm{mt}}$ (green) on the mitoribosome. $\mathbf{c} \ln \mathrm{EF}-\mathrm{G} 1_{\mathrm{mt}}{ }^{6}, \mathrm{the}$ presence of two universally conserved glycine residues (dark green) at the tip of domain IV loop1 region (salmon) facilitates its insertion between the mRNA-tRNA duplex at the decoding center (DC) during EF-G1 $\mathrm{mt}_{\mathrm{t}}$-catalyzed translocation. $\mathbf{d}$ In EF-G2 $2_{\mathrm{mt}}$, the second glycine of domain IV loop 1 region (red) is substituted by an aspartic acid (dark green) altering its conformation and flexibility, and thereby making its insertion into the DC during translocation unfavorable. e The boxed aa sequence corresponds to the loop 1 situated at the tip of domain IV, which is conserved between EF-G1 $\mathrm{mt}$ and the T. thermophilus EF-G. First of the two universally conserved loop 1 glycine residues (green) is substituted by an alanine in B. burgdorferi EF-G2, while the second one is replaced by an aspartic acid in $\mathrm{EF}-\mathrm{G} 2_{\mathrm{mt}}$.

mRNA-tRNA duplex with the universally conserved 16S rRNA bases A1492 and A1493 in the 30S A site thereby aiding the A-site tRNA along with its associated codon to translocate into the $\mathrm{P}$ site $^{67}$. The loop 1 region is conserved in EF-G1 $1_{m t}$ but is significantly altered in EF-G2 ${ }_{\mathrm{mt}}$ (Fig. 6e). EF-G1 $1_{\mathrm{mt}}$ retains both the glycine residues (Gly544 and Gly545) in its domain IV loop 1 region (Fig. 6c) whereas the second glycine is replaced by an aspartic acid in EF-G2 $2_{\mathrm{mt}}$ (Fig. 6d), which alters the conformation of the tip of domain IV rendering it structurally unfavorable for insertion into the grove between the P-site tRNA and the associated codon (Fig. 6d). A similar observation has been made in case of the spirochaete $B$. burgdorferi that utilizes EF-G2 exclusively for ribosome recycling 42 , where one of these conserved loop1 glycines is substituted by an alanine (Fig. 6e) rendering it inactive for promoting the translocation step. Interestingly, however, the T. thermophilus EF-G2 carries both the conserved glycines. Moreover, the Ala546 and Gly547 residues that follow the conserved glycines of loop 1 in EF$\mathrm{G} 1_{\mathrm{mt}}$ are substituted by the large polar sidechain residues lysine and arginine, respectively, in the corresponding region of EF$\mathrm{G} 2 \mathrm{mt}_{\mathrm{m}}$ (Fig. 6e), thereby significantly altering the hydrophobicity of the loop 1 tip. Point mutations and deletions at the loop 1 tip region are known to have a pronounced effect on the function of EF-G during the elongation step of bacterial protein synthesis ${ }^{68}$, but have a negligible effect on the activity of EF-G in bacterial ribosome disassembly ${ }^{69}$. Our study thus provides a structural rationale for the biochemical finding that mammalian mitoribosomes utilize two distinct EF-G-like factors during the translation elongation and recycling phases ${ }^{27}$.

Mammalian 55S mitoribosome recycling does not require GTP hydrolysis by EF-G2 ${ }_{\text {mt. }}$. The overall $G$ domain structure in the $39 \mathrm{~S} \bullet \mathrm{RRF}_{\mathrm{mt}} \bullet \mathrm{EF}-\mathrm{G} 2_{\mathrm{mt}}$ complex (Class III map) is similar to the G domain in the $55 \mathrm{~S} \cdot E F-G 1_{m t}$ complex $^{6,46}$. The well-ordered density in our maps of highly conserved translational GTPase consensus motifs such as the P-loop, switch I and switch II regions, allowed complete modeling of these essential regions. A welldefined density corresponding to a bound GMPPCP molecule is also readily identifiable in the nucleotide binding pocket. GMPPCP is stabilized through interactions with universally conserved aa residues, such as Asp80 and Lys83 of P-loop, Thr122 of switch I and His145 of switch II (Fig. S8a). As in the $55 \mathrm{~S} \bullet \mathrm{EF}-\mathrm{G} 1_{\mathrm{mt}}$ complex ${ }^{6}$, a crucial $\mathrm{Mg}^{2+}$ ion is positioned near the $\gamma$ phosphate of GMPPCP and is coordinated by Thr84 and Thr122 from the P-loop and switch I regions, respectively (Fig. S8a). The catalytic His145 (His124 in EF-G1 $1_{\mathrm{mt}}$ ), known to play a central role in the hydrolysis of the bound nucleotide ${ }^{70,71}$, is found oriented towards the $\gamma$ phosphate of the bound GMPPCP (Fig. S8a), suggesting an active conformation of the factor prior to GTP hydrolysis.

The highly conserved $\alpha$-sarcin-ricin stem-loop (SRL) region is known to be essential for the GTPase activity of all the translational $G$ proteins ${ }^{47,70,71}$. Base A3129 from the SRL was 

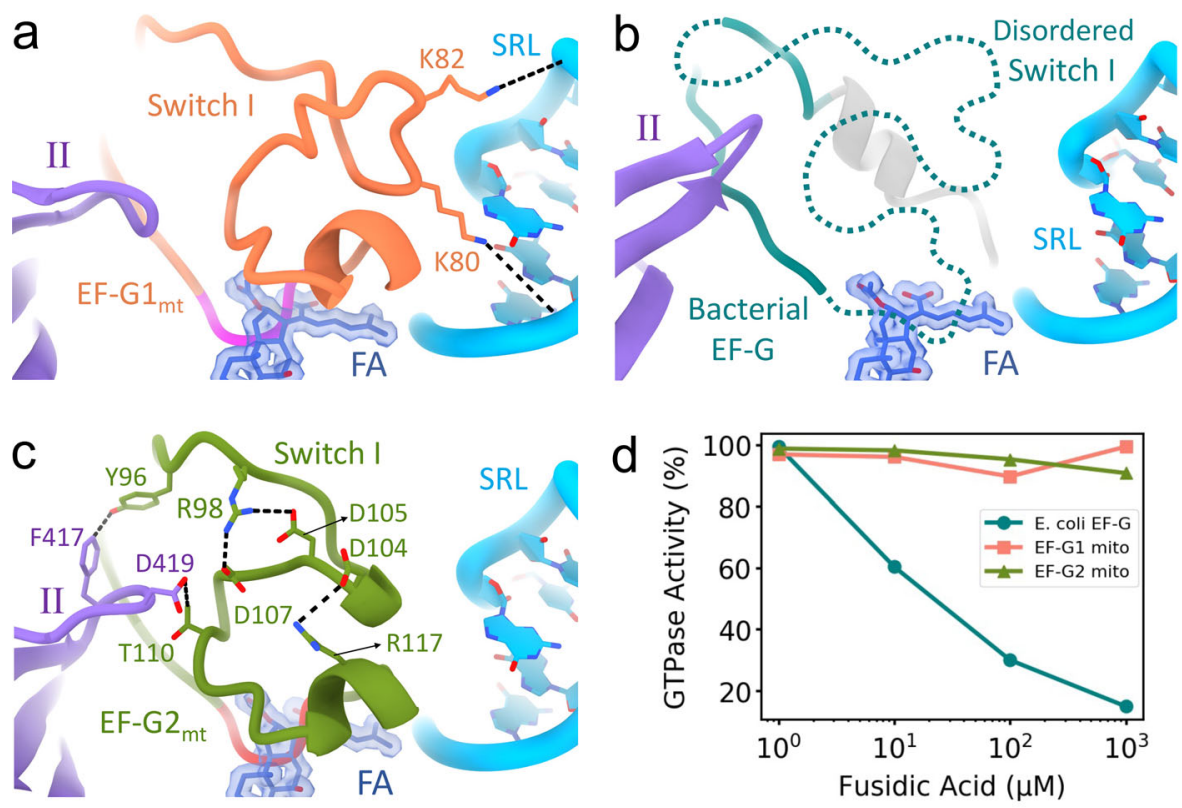

e $\begin{array}{lll}\text { EF-G(Tt): } & \text { YYTGRIHKIGEVHE---GAATMDFMEQERERGITITAAVTTC } & 072 \\ \text { EF-GImt: } & \text { YYTGRIAKMHEVKGKDGVGAVMDSMELERQRGITIQSAATYT } & 109 \\ \text { EF-G2mt: } & \text { YYSGYTRSLGDVDD---GDTVTDFMAQERERGITIQSAAVTF } & 130\end{array}$

Fig. 7 EF-G1 $1_{m t}$ and EF-G2 ${ }_{m t}$ follow diverse mechanisms to render resistance to the antibiotic fusidic acid (FA). a Stabilized Switch I region (salmon) ${ }^{6}$ in $\mathrm{EF}-\mathrm{G} 1_{\mathrm{mt}}$ blocks FA (dark blue) from accessing its binding site. The Switch I region sterically overlapping with FA is colored in magenta. Switch I stability is achieved by the presence of two unique lysine residues (K80 and K82) that strongly interact with the phosphates of SRL by forming salt bridges (light blue ${ }^{46}$. b The absence of these lysine residues in the Switch I region (dark cyan) of bacterial EF-G disorders the Switch $1^{47}$ and hence makes it susceptible to FA binding and inhibition. $\mathbf{c}$ In EF-G2 $2_{\mathrm{mt}}$, the primary aa sequence of switch I (green) is highly altered enabling the formation of three salt bridges within its switch I, thereby stabilizing it. Furthermore, strong interactions are observed between the switch I and domain II (purple) in EF-G2 $2_{\text {m. }}$ The Switch I region sterically overlapping with $F A$ is colored in red. $\mathbf{d} E F-G 1_{m t}$ (salmon) and EF-G2 $2_{m t}$ (green) show strong resistance even at high concentrations of FA, while the bacterial EF-G (dark cyan) is susceptible to FA inhibition even at low concentrations. Each point in the plots represents the average of normalized data from two independent sets of experiments. Raw luminescence values obtained from the GTPase-Glo ${ }^{\text {TM }}$ Assay are shown in Supplementary Table 1. e The aa sequence alignment of the switch I region (boxed) in three EF-Gs. The lysine residues shown in salmon are unique to mammalian EF-G1 $\mathrm{mt}$ and confer stability to switch I by interacting with the SRL. Residues shown in green (except R117) are unique to EF-G2 $2_{\mathrm{mt}}$ and confer stability to switch I by forming salt bridges within the switch I region.

found to be contacting the Switch II His145 through hydrogen bonding interactions and thereby stabilizing His145 in its activated conformation poised to perform the hydrolysis reaction (Fig. S8b). While EF-G-dependent GTP hydrolysis is essential for an efficient splitting of the 70 S ribosome into its subunits ${ }^{16,18,22}$, dissociation of the mammalian 55S mitoribosomes does not require EF-G2 ${ }_{\mathrm{mt}}$-dependent GTP hydrolysis, which is only needed for the release of EF-G $2_{\mathrm{mt}}$ from the dissociated large subunit ${ }^{27}$. Our structural results strongly corroborate the Tsuboi and coworkers ${ }^{27}$ observation as a significant proportion (82\%) of the EF-G2 $\mathrm{mt}$ was found complexed to the $39 \mathrm{~S}$ subunits as compared to the small proportion (18\%), that remained associated with the $55 \mathrm{~S}$ mitoribosomes (see Fig. S1). Even though GTP hydrolysis by EF-G2 $2_{\mathrm{mt}}$ is not necessary for $55 \mathrm{~S}$ mitoribosome splitting, the presence of GTP or its non-hydrolysable analogs GDPNP/GMPPCP in the nucleotide binding pocket is essential, as the presence of GDP or the absence of any nucleotide does not split the $55 \mathrm{~S}$ mitoribosome ${ }^{27}$.

Divergent mechanisms of fusidic acid (FA) resistance by EF$\mathbf{G} \mathbf{1}_{\mathbf{m t}}$ and EF-G2 $\mathbf{m t}_{\mathbf{m}}$. FA is a fusidane class antibiotic that is used to treat bacterial skin infections along with chronic bone and joint infections. It is effective against several species of gram-positive bacteria and is clinically used to treat methicillin-resistant $S$. aureus (MRSA). FA prevents the release of EF-G•GDP from the
70 S ribosome after GTP hydrolysis by preventing the switch II from attaining its GDP-bound conformation ${ }^{47}$. Prior structural studies have demonstrated that FA binds in an interdomain pocket between the G domain, domain II, and domain III of EF$\mathrm{G}^{47,72}$. Stable binding of FA requires the switch I region to be disordered $^{47}$, because an ordered switch I would overlap with the binding site of FA. Biochemical studies have shown that a substantially higher concentration (10-fold to 100 -fold) of FA is needed to inhibit the activity of EF-G1 $1_{\mathrm{mt}}$ during mitochondrial elongation ${ }^{28,73}$. Recent cryo-EM structures of EF-G1 $1_{\mathrm{mt}}$ bound to the 55S mitoribosomes have presented the switch I in a welldefined conformation ${ }^{6,46}$ (Fig. 7a), in contrast to bacterial 70S•EF-G complexes where the density for switch I has been consistently poorly resolved (Fig. $7 \mathrm{~b})^{47,67,70-72}$. It was proposed that the increased resistance observed for EF-G1 $1_{\mathrm{mt}}$ towards FA resulted from a small insertion in the switch I region of EF$\mathrm{G} 1_{\mathrm{mt}}{ }^{46}$. The two positively charged lysine residues (Lys80 and Lys82) in this insertion form salt bridges with the negatively charged phosphate backbone of the SRL from the 39S subunit (Fig. 7b), and hence were hypothesized to confer additional stability to the switch I of EF-G $1_{\mathrm{mt}}{ }^{46}$. Biochemical evidence showed EF-G $1_{\mathrm{mt}}$ to be more resistant towards FA than its bacterial counterpart 28,73 .

FA is known to inhibit both the translocation and the ribosome recycling steps in bacteria, though there is conflicting evidence on which step of translation is primarily targeted by $\mathrm{FA}^{22,25}$. 


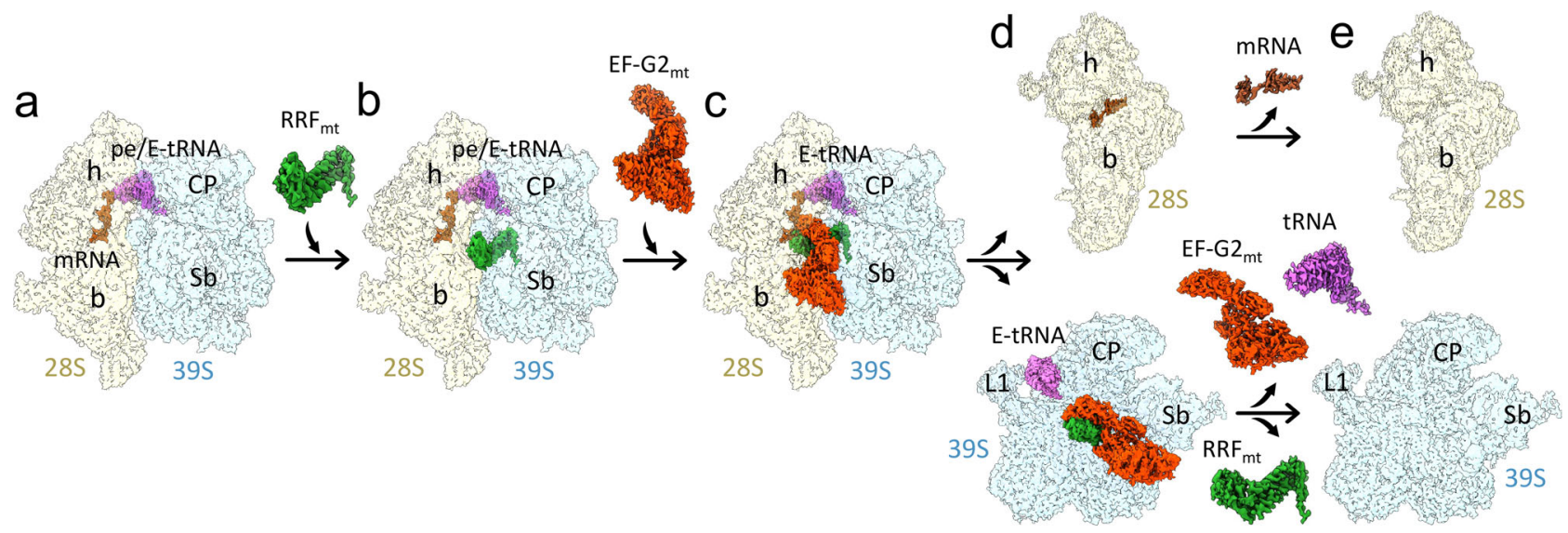

Fig. 8 Sequence of main events in human mitochondrial ribosome recycling suggested by structures in this study. a The model post-termination

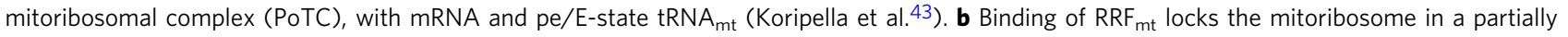
destabilized state with rotated $28 \mathrm{~S}$ subunit (Figs. 1a and S3b). The mito-specific N-terminus of the RRF $\mathrm{mt}^{\prime} \mathrm{S}$ NTE occupies a unique site near the bridge B2a region (Fig. 2). c Subsequent binding of EF-G2 $\mathrm{mt}$ further destabilizes the $55 \mathrm{~S}$ complex with disruption of additional inter-subunit bridges, in a fast reaction leading to multiple short-lived intermediate states, with the $28 \mathrm{~S}$ subunit present in multiple orientations relative to the $39 \mathrm{~S}$ subunit (Fig. 1b). $\mathbf{d} 28 \mathrm{~S}$ and $39 \mathrm{~S}$ subunits are dissociated. While the $28 \mathrm{~S}$ subunit still carries mRNA, the $39 \mathrm{~S}$ subunit carries $\mathrm{RRF}_{\mathrm{mt}}, \mathrm{EF}-\mathrm{G} 2_{\mathrm{mt}}$, and surprisingly a deacylated tRNA $\mathrm{A}_{\mathrm{mt}}$ in the $39 \mathrm{~S}$ subunit's E-site region (Figs. 1c and 4). This is in sharp contrast to the tRNA dissociation mechanism in eubacterial ribosome recycling, where tRNA goes with the small $30 \mathrm{~S}$ ribosomal subunit. e Depiction of final recycling products, steps of ligand release between $\mathbf{d}$ and $\mathbf{e}$ are yet to be characterized.

Comparison of the FA binding pocket between the bacterial EF$\mathrm{G}, \mathrm{EF}-\mathrm{G} 1_{\mathrm{mt}}$, and EF-G2 $2_{\mathrm{mt}}$ revealed that key aa residues (Fig. S9a) reported to be necessary for the stable binding of $\mathrm{FA}^{47,72}$ are highly conserved (Fig. S9c), thereby suggesting a similar binding mechanism for FA for all the three EF-Gs. The three aa insertion in $\mathrm{EF}-\mathrm{G} 1_{\mathrm{mt}}$ that confers resistance to $\mathrm{FA}$ is not present in EF$\mathrm{G} 2 \mathrm{mt}_{\mathrm{m}}$ and the corresponding region in EF-G2 $2_{\mathrm{mt}}$ does not contain any positively charged aa residues (Fig. 7e), that could strongly interact with the SRL. However, the cryo-EM map of EF-G2 shows a well-resolved density for the switch I region (Fig. S9b) and enabled its modeling (Fig. 7c), indicating an alternative mechanism for the stabilization of switch I in EF-G2 ${ }_{\mathrm{mt}}$. Sequence alignment showed that the switch I region composition in EF$\mathrm{G} 2_{\mathrm{mt}}$ is significantly different as compared to EF-G1 $1_{\mathrm{mt}}$ and the bacterial EF-G (Fig. 7e). Three new salt bridge interactions were identified within the switch I of EF-G2 ${ }_{\mathrm{mt}}$. Arg98 forms the first two salt bridges by pairing with Asp105 and Asp107, respectively, while Arg117 and Asp104 are involved in the formation of the third salt bridge (Fig. 7c). Furthermore, stronger interactions are observed between the switch I and domain II in EF-G2 compared to EF-G1 $1_{\mathrm{mt}}$. Thr110 from switch I is placed in the close proximity of Asp419 of domain II with the possibility of hydrogen bond formation (Fig. 7c), while the corresponding interaction in EF-G1 $1_{\mathrm{mt}}$ is between Asp404 and Val88, a much weaker interaction with no possibility of hydrogen bond formation. There is also potential for a tight T-stacking interaction between Phe417 and Tyr96 in EF-G2 ${ }_{\mathrm{mt}}$ (Fig. 7c), while the corresponding residues in EF-G1 $1_{\mathrm{mt}}$ being His402 and Arg72 offer no such interaction. Overall, through a combination of internal salt bridges and additional contacts with domain II, switch I gets highly stabilized in EF-G2 ${ }_{\mathrm{mt}}$. Since a stabilized switch I region occludes the binding site of FA (Fig. 7a, b), EF$\mathrm{G} 2{ }_{\mathrm{mt}}$ is expected to exhibit strong resistance towards FA in the lines of EF-G1 $1_{\mathrm{mt}}$. To test this hypothesis, we measured the GTPase activity of EF-G $2_{\mathrm{mt}}$ alongside EF-G1 $1_{\mathrm{mt}}$ and E. coli EF-G in the presence of FA under multiple-turnover conditions (see Methods). Our data shows that while $1 \mu \mathrm{M}$ FA has no effect on the GTPase activity in E. coli, significant inhibition is observed at higher concentrations of FA. In contrast, FA has almost no effect on the GTPase activity of either EF-G1 $1_{\mathrm{mt}}$ or EF-G2 $2_{\mathrm{mt}}$ even up to
$10 \mathrm{mM}$ FA. Our results are consistent with the earlier finding that EF-G1 $1_{\mathrm{mt}}$ is highly resistant to FA compared to the bacterial EF$\mathrm{G}^{28,73}$, and also consistent with recent finding that showed EF$\mathrm{G} 22_{\mathrm{mt}}$ is not susceptible to inhibition by $\mathrm{FA}^{74}$. Our results and analysis suggest FA resistance in EF-G2 ${ }_{\mathrm{mt}}$ occurs by a structural mechanism fundamentally different from that in EF-G1 $1_{\mathrm{mt}}$.

In conclusion, structures of three distinct functional states formed during the process of human mitoribosome recycling are presented (Fig. 1). A previous biochemical finding that GTP hydrolysis is not required for the $\mathrm{RRF}_{\mathrm{mt}} \cdot \mathrm{EF}-\mathrm{G} 2_{\mathrm{mt}}$-mediated splitting of the post-termination mitoribosomal complex is corroborated. We also show that a mito-specific segment of the $\mathrm{RRF}_{\mathrm{mt}}$ 's NTE compensates for the slightly reduced size of the H69 within the 16S rRNA of the mitoribosomal large subunit (Fig. 2). This is the first evidence showing a translational factor compensating for an rRNA segment lost, perhaps during the evolution of the mammalian mitochondrial translation machinery. Our structures reveal how $\mathrm{RRF}_{\mathrm{mt}}$ 's domain II and EF-G2 domain IV directly help in disrupting the central inter-subunit bridge, B2a (Fig. 3), how the deacylated tRNA is held with the dissociated 39S subunit components (Fig. 4), and suggest how the dynamics of the interactions among the uL11m, CTD of $\mathrm{uL} 12 \mathrm{~m}$, and the G'domain of EF-G2 $2_{\mathrm{mt}}$ alters between elongation and recycling steps (Fig. 5). Structural analysis of domain IV of EF$\mathrm{G} 1_{\mathrm{mt}}$ and EF-G2 $\mathrm{mt}$ explains their specific roles in two distinct steps of elongation and recycling, respectively (Fig. 6). Analysis of their GTPase domains complemented by GTPase assays reveal two distinct mechanisms of antibiotic fusidic acid resistance adopted by two homologous GTPases (Fig. 7). These observations allow us to highlight distinct features of the main steps of human mitoribosomal recycling (Fig. 8). Future studies using timeresolved cryo-EM should help further resolve the short-lived intermediates that form during the transition from Class I to Class III states.

\section{Methods}

Purification of $\mathbf{5 5 S}$ mitoribosomes. The source of mitochondrial ribosomes was human embryonic kidney cells lacking $\mathrm{N}$-acetyl-glucosaminyltransferase I (HEK293S GnTI) that were cultured in roller bottles using FreeStyle ${ }^{\mathrm{TM}} 293$ media (Gibco, Life Technologies) supplemented with $5 \%$ fetal bovine serum (Gibco, Life 
Technologies). After centrifugation at $1000 \times g$ for $7 \mathrm{~min}$, the HEK293S GnTI cellpellet was transferred to a glass homogenizer and resuspended in buffer containing $50 \mathrm{mM}$ HEPES-KOH pH 7.5, $10 \mathrm{mM}$ KCL, $1.5 \mathrm{mM}$ MgOAc, $70 \mathrm{mM}$ sucrose, 210 $\mathrm{mM}$ mannitol, $1 \mathrm{mM}$ EDTA, $1 \mathrm{mM}$ EGTA, $1 \mathrm{mM}$ DTT, and $1 \mathrm{mM}$ PMSF. The cells were homogenized by applying 120 strokes and the supernatant was separated from the cell debris by spinning at $950 \times g$ for $15 \mathrm{~min}$. The supernatant was then spun at $11,000 \times g$ for $15 \mathrm{~min}$, and the resulting pellet that contains crude mitochondria was resuspended in SEM buffer $(250 \mathrm{mM}$ sucrose, $20 \mathrm{mM}$ HEPES-KOH $\mathrm{pH} 7.5,1 \mathrm{mM}$ EDTA, and $1 \mathrm{mM}$ EGTA). RNase-free DNase $\mathbf{I}$ ( 3 units/ml) was added to the crude mitochondria and incubated at $4{ }^{\circ} \mathrm{C}$ for $1 \mathrm{~h}$ in a rocking platform to allow gentle mixing. A discontinuous gradient was prepared in a Beckman polyallomer tube by layering $2.5 \mathrm{ml}$ of $60 \%, 4 \mathrm{ml}$ of $32 \%, 1 \mathrm{ml}$ of $23 \%$, and $1 \mathrm{ml}$ of $15 \%$ sucrose solutions in buffer containing $10 \mathrm{mM}$ HEPES-KOH pH 7.5 and $1 \mathrm{mM}$ EDTA. DNase-treated sample was loaded on the discontinuous gradient and centrifuged for $1 \mathrm{~h}$ at 135,000 $\times g$ using Ti70 rotor in Beckman ultracentrifuge. The brownish-orange layer containing pure mitochondria was carefully separated and re-suspended in SEM buffer.

Four volumes of lysis buffer ( $25 \mathrm{mM}$ HEPES-KOH pH 7.5, $100 \mathrm{mM} \mathrm{KCl,} 25$ $\mathrm{mM} \mathrm{MgOAc}, 1.7 \%$ Triton X-100, $2 \mathrm{mM}$ DTT and $1 \mathrm{mM}$ PMSF) was added to the mitochondrial-pellet and then incubated for $15 \mathrm{~min}$ at $4{ }^{\circ} \mathrm{C}$. The sample was centrifuged at 30,000 $\times g$ for $20 \mathrm{~min}$ and the supernatant was loaded on top of $1 \mathrm{M}$ sucrose cushion in buffer (20 mM HEPES-KOH pH 7.5, $100 \mathrm{mM} \mathrm{KCl}, 20 \mathrm{mM}$ $\mathrm{MgOAc}, 1 \%$ Triton X-100 and $2 \mathrm{mM}$ DTT). After centrifugation for $17 \mathrm{~h}$ at 90,000 $\times g$ using Ti70 rotor in Beckman ultracentrifuge, a minimal volume of Mitobuffer (20 mM HEPES-KOH pH 7.5, $100 \mathrm{mM} \mathrm{KCl}, 20 \mathrm{mM} \mathrm{MgOAc}$, and $2 \mathrm{mM}$ DTT) enough to dissolve the pellet was added. $10-30 \%$ continuous sucrose density gradients were prepared in Mitobuffer, using the gradient making apparatus (C.B.S. Scientific Co.). The resuspended pellet was subjected to $10-30 \%$ continuous sucrose density gradient centrifugation at $60,000 \times g$ for $17 \mathrm{~h}$ using Sw32 rotor in Beckman ultracentrifuge. The gradient was fractionated on ISCO gradient analyzer (Teledyne ISCO, Inc), and the fractions corresponding to the mitoribosomes were collected and pooled. Finally, the pooled mitoribosomes were concentrated by spinning them at $130,000 \times g$ for $6 \mathrm{~h}$ using Ti70 rotor, and the pellet was resuspended in Polymix buffer ( $5 \mathrm{mM}$ HEPES-KOH pH 7.5, $100 \mathrm{mM} \mathrm{KCl}, 20 \mathrm{mM} \mathrm{MgOAc}, 5 \mathrm{mM}$ $\mathrm{NH}_{4} \mathrm{Cl}, 0.5 \mathrm{mM} \mathrm{CaCl}_{2}, 1 \mathrm{mM}$ DTT, $1 \mathrm{mM}$ spermidine, and $8 \mathrm{mM}$ putrescine).

Overexpression and purification of $\mathbf{R R F 2} \mathbf{m}_{\mathbf{m t}} \cdot \mathrm{RRF}_{\mathrm{mt}}$ clone was a gift from Prof Linda Spremulli, University of North Carolina. The SUMO-tagged $\mathrm{RRF}_{\mathrm{mt}}$ was over-expressed in Rosetta2 cell lines and lysis buffer $(500 \mathrm{mM} \mathrm{NaCl}, 1 \times \mathrm{PBS}, 4 \mathrm{mM}$ B-mercaptoethanol, $1 \mathrm{mM}$ PMSF, and $10 \mathrm{mM}$ imidazole) was added to the pelleted cells. After sonication, the lysate was treated with DNase and then centrifuged for $30 \mathrm{~min}$ at $16,000 \times g$. The supernatant was applied to a His-trap $\mathrm{Ni}^{2+}$ column and the SUMO-tagged protein was eluted from the column using elution buffer $(250$ $\mathrm{mM} \mathrm{NaCl}, 1 \times$ PBS, $4 \mathrm{mM}$ ß-mercaptoethanol, and $300 \mathrm{mM}$ imidazole) using standard affinity purification protocols. The purified protein was dialyzed in buffer (20 mM Tris- $\mathrm{HCl} \mathrm{pH} \mathrm{8.0,250} \mathrm{mM} \mathrm{NaCl,} 4 \mathrm{mM} \mathrm{B-mercaptoethanol,} \mathrm{and} \mathrm{5 \%} \mathrm{gly-}$ cerol) and then the SUMO tag was cleaved from $\mathrm{RRF}_{\mathrm{mt}}$ by incubation with SUMO express protease for $1 \mathrm{~h}$ at $30^{\circ} \mathrm{C}$. Finally, the SUMO tag and the SUMO express protease were separated from the $\mathrm{RRF}_{\mathrm{mt}}$ by passing through a His-trap $\mathrm{Ni}^{2+}$ column that specifically adsorbs the SUMO tag and the SUMO express protease while pure $\mathrm{RRF}_{\mathrm{mt}}$ was released into the column flow-through.

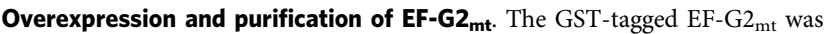
cloned into pGEX6.1 vector and over-expressed in Rossetta (pLysS + RARE) cell lines. Cells were grown in LB media with $100 \mu \mathrm{g} / \mathrm{ml}$ ampicillin until 0.6 O.D. and protein over-expression was induced by adding $100 \mathrm{mM}$ isopropyl-1-thio-Dgalactopyranoside (IPTG). The cell culture was left overnight at $16^{\circ} \mathrm{C}$ for optimal protein yields. The cells were pelleted in a JLA 10.5 rotor at $5000 \mathrm{rpm}$ for $30 \mathrm{~min}$ and were shock-frozen in liquid nitrogen and stored at $-80^{\circ} \mathrm{C}$. The frozen cells were resuspended in lysis buffer (1× PBS-pH7.5, $10 \mathrm{mM} \mathrm{MgCl}_{2}, 1 \mathrm{mM}$ phenylmethyl sulphonyl fluoride (PMSF) and $1 \mathrm{mM}$ DTT). After sonication and DNase I $(5 \mu \mathrm{g} / \mathrm{ml})$ treatment, the lysate were centrifuged at $14,000 \mathrm{rpm}$ for $30 \mathrm{~min}$ to remove cell debris. The supernatant was passed through GSTrap ${ }^{\text {ww }}$ HP column equilibrated with binding buffer $1 \times$ PBS-pH7.5 and 1 mM DTT. The GST-tag was cleaved from EF-G2 $2_{\mathrm{mt}}$ by loading $3 \mathrm{C}$ precision protease (gifted by Dr. Hongmin Li's lab, Wadsworth center, USA) along with cleavage buffer (50 mM Tris-HCl-pH $7.5,150 \mathrm{mM} \mathrm{NaCl}$, and $1 \mathrm{mM}$ DTT). After incubating the EF-G2 ${ }_{\mathrm{mt}}$ with $3 \mathrm{C}$ precision protease overnight, relatively pure $\mathrm{EF}-\mathrm{G} 2_{\mathrm{mt}}$ was eluted by passing the elution buffer (50 mM Tris-HCl-pH 7.5, $150 \mathrm{mM} \mathrm{NaCl}$ and $1 \mathrm{mM} \mathrm{DTT}$ ). To obtain highlevel purity, EF-G2 ${ }_{\mathrm{mt}}$ was further passed through an anion exchange HiTrap Q HP column (GE healthcare, USA) and pure protein was eluted by running a gradient with $20 \mathrm{mM}$ Tris-HCl-pH 7.5 and $500 \mathrm{mM} \mathrm{NaCl}$.

Preparation of the 55S•RRF ${ }_{m t} \bullet E_{F}-\mathbf{G}_{\mathrm{mt}} \bullet \mathbf{G M P P C P}$ complex. Fifty micromolar of puromycin and $150 \mathrm{nM} 55 \mathrm{~S}$ mitoribosomes were mixed in HEPES polymix buffer $\left(5 \mathrm{mM}\right.$ HEPES-KOH pH 7.5, $100 \mathrm{mM} \mathrm{KCl}, 20 \mathrm{mM} \mathrm{MgOAc}, 5 \mathrm{mM} \mathrm{NH}_{4} \mathrm{Cl}, 0.5 \mathrm{mM}$ $\mathrm{CaCl}_{2}, 1 \mathrm{mM}$ DTT, $1 \mathrm{mM}$ spermidine, and $8 \mathrm{mM}$ putrescine) and incubated for 10 min at $37^{\circ} \mathrm{C}$ to obtain the model post-termination (PoTC) complex. Fifteen micromolar of $\mathrm{RRF}_{\mathrm{mt}}$ was added to this reaction mixture of PoTC and incubated for an additional $5 \mathrm{~min}$ at $37^{\circ} \mathrm{C}$ to obtain the $55 \mathrm{~S} \cdot \mathrm{RRF}_{\mathrm{mt}}$ complex as described earlier ${ }^{7}$. Five micromolar of EF-G2 $2_{\mathrm{mt}}$, together with $200 \mu \mathrm{M}$ GMPPCP, was added to the $55 \mathrm{~S} \cdot \mathrm{RRF}_{\mathrm{mt}}$ complex and incubated for various timepoints $(5 \mathrm{~s}, 30 \mathrm{~s}$ and 2 $\mathrm{min}$ ) at $37^{\circ} \mathrm{C}$ to obtain the $55 \mathrm{~S} \cdot \mathrm{RRF}_{\mathrm{mt}} \cdot \mathrm{EF}-\mathrm{G} 2_{\mathrm{mt}} \cdot{ }^{\circ} \mathrm{GMPPCP}$ complex, which was immediately utilized for the cryo-EM grid preparation.

GTPase-Glo assay. GTPase activity was measured using the GTPase-Glo ${ }^{\text {mo }}$ Assay by Promega and carried out as described ${ }^{75}$. In brief, a $10 \mu \mathrm{l}$ reaction consisting of $0.1 \mu \mathrm{M}$ ribosomes, $0.25 \mu \mathrm{M}$ either bacterial (E. coli) EF-G or EF-G1 $1_{\mathrm{mt}}$ or EF-G2 $2_{\mathrm{mt}}$ and $1 \mu \mathrm{M}$ GTP were incubated at room temperature in GTPase-Glo ${ }^{\mathrm{mt}}$ buffer in the absence and presence of varying amounts of fusidic acid (FA) for $1 \mathrm{~h}$. Ten microliter of Reconstituted GTPase-Glo ${ }^{m b}$ reagent was added to the reaction and left shaking at room temperature for $30 \mathrm{~min}$. Finally, $20 \mu \mathrm{L}$ of detection reagent was added, incubated for $10 \mathrm{~min}$ at room temperature and luminescence was measured in a Turner Biosystems Veritas ${ }^{\mathrm{ww}}$ Microplate Luminometer after $10 \mathrm{~min}$. A negative control that contained only $1 \mu \mathrm{M}$ GTP and a positive control that contained no FA were used each time. Luminescence was measured at FA concentrations of 1, 10, 100 , and $1000 \mu \mathrm{M}$. Data was collected in duplicates and each duplicate dataset was normalized between 0 and 1 using the equation:

$$
x_{\text {norm }}=\frac{x-x_{\min }}{x_{\max }-x_{\min }}
$$

As GTPase activity and luminescence measured had an inverse relationship, \% GTPase activity was calculated with the formula \%GTPase activity $=\left(1-x_{\text {norm }}\right) \times$ 100. The values at each point were then averaged and standard deviation was calculated. The raw luminescence values are presented in Table S1. The plot was generated using Python library Matplotlib.

Cryo-electron microscopy and image processing. Home-made carbon was coated as a continuous layer ( $\sim 50 \AA$ thick) onto Quantifoil holey carbon $1.2 / 1.3$ copper grids, which were then glow-discharged for $30 \mathrm{~s}$ on a plasma sterilizer. Four microliter of the sample was loaded to each of the multiple grids, incubated for $15 \mathrm{~s}$ at $4{ }^{\circ} \mathrm{C}$ and $100 \%$ humidity, and then blotted for $4 \mathrm{~s}$ before flash-freezing into the liquid ethane using a Vitrobot IV (FEI). Data was acquired on a Titan Krios electron microscope equipped with a Gatan K2 summit direct-electron detecting camera at $300 \mathrm{KV}$. A defocus range of -1.0 to $-3.0 \mu \mathrm{m}$ was used at a calibrated magnification of $22,500 \times$, yielding a pixel size of $1.0732 \AA$ on the object scale. A dose rate of 8.25 electrons per pixel per second and an exposure time of 10 seconds resulted in a total dose of $71.6 \mathrm{e}^{-2}$. CryoSPARC 3.0.1 $1^{76}$ was employed for all the subsequent data processing steps. After applying full-frame motion correction to al 50 movie frames corresponding to each of the 21,752 micrographs that were collected, 142 bad micrographs were deselected after determining their contrast transfer function (CTF) using CTFFIND4 ${ }^{77}$. From the remaining 21,610 micrographs, a total of 5,046,906 particles were autopicked, which were then subjected to local motion correction and then 4,049,952 particles were retained. This step was followed by reference-free $2 \mathrm{D}$ classification which allowed us to further separate the good particles $(1,140,751)$ from the bad particles $(2,909,201)$ based on the $2 \mathrm{D}$ averages. Reference-based 3D classification was employed first to separate the particles into intact $55 \mathrm{~S}$ mitoribosomes (162,354 particles), 39S subunits $(419,699$ particles), $28 \mathrm{~S}$ subunits (235,896 particles) and poorly aligned particles $(322,802$ particles). To obtain more homogenous sub-populations, particles corresponding to the 55S mitoribosomes, $39 \mathrm{~S}$ and $28 \mathrm{~S}$ subunits were each subjected to additional rounds of $3 \mathrm{D}$ classification that finally yielded three stable classes representing three distinct functional states formed during the human mitoribosome recycling process. Two $55 \mathrm{~S}$ mitoribosome classes, Class I (93,212 particles) and Class II (28,929 particles), were finally refined to 3.49 and $3.91 \AA$ A, respectively, while the $39 \mathrm{~S}$ subunit Class III (132,008 particles) was refined to $3.15 \AA$.

Model building and optimization. Coordinates corresponding to the small and large subunits from our published human mitoribosome structures ${ }^{6}$ (PDB ID: 6VLZ) were docked independently as rigid bodies into the corresponding cryo-EM density maps of the Class I, Class II, and Class III complexes using Chimera 1.14 (Pettersen EF 2004). To obtain optimal fitting into our cryo-EM densities, the models were subsequently refined in PHENIX 1.14 (Adams PD 2010) using the "real-space refinement" function. Coordinates belonging to the human $\mathrm{RRF}_{\mathrm{mt}}$ (Koripella et $\mathrm{al} .{ }^{43}$ ) were placed independently into the corresponding cryo-EM densities of all the three maps as rigid bodies using Chimera $1.14^{78}$ and the models were further real-space refined in PHENIX $1.14^{79}$ to achieve optimal accommodation into the cryo-EM densities. The primary aa sequence of EF-G2 $2_{\mathrm{mt}}$ was submitted to the I-TASSER server ${ }^{80}$ to generate the initial EF-G2 ${ }_{\mathrm{mt}}$ homology model that was used to interpret the corresponding cryo-EM density. Segments in the homology model that do not fully accommodate into the corresponding EF$\mathrm{G} 2 \mathrm{mt}$ density were modeled de novo using Chimera $1.14^{78}$ and COOT 0.9.5 $5^{81}$. For the final optimization of the model into the cryo-EM density, the "Real-space refinement" function in PHENIX $1.14^{79}$ was utilized. Validation reports for all the models were obtained from the Molprobity server ${ }^{82}$ and the overall statistics of EM reconstruction and molecular modeling are listed in Table S2. All the figures in the manuscript were generated using ChimeraX 1.1.183. 
Reporting summary. Further information on research design is available in the Nature Research Reporting Summary linked to this article.

\section{Data availability}

The cryo-EM density maps and the atomic coordinates have been deposited in the Electron Microscopy Data Bank and the Protein Data Bank under the accession codes EMD-23096 and PDB ID 7L08 for the $\mathrm{RRF}_{\mathrm{mt}}$-bound human 55S mitoribosome (Complex I), EMD-23114 for the $\mathrm{RRF}_{\mathrm{mt}} \cdot \mathrm{EF}-\mathrm{G}_{\mathrm{mt}}$-bound human $55 \mathrm{~S}$ mitoribosome (Complex II), EMD-23121 [https://www.ebi.ac.uk/pdbe/entry/emdb/EMD-23121] and PDB ID 7L20 for the $\mathrm{RRF}_{\mathrm{mt}} \bullet \mathrm{EF}-\mathrm{G} 2_{\mathrm{mt}}$-bound human $39 \mathrm{~S}$ mitoribosome (Complex III). All the raw image stacks used in the $3 \mathrm{D}$ reconstructions are being made available through EMPIAR [https://www.ebi.ac.uk/pdbe/emdb/empiar/entry/10703].

Received: 21 January 2021; Accepted: 14 May 2021; Published online: 14 June 2021

\section{References}

1. Pel, H. J. \& Grivell, L. A. Protein synthesis in mitochondria. Mol. Biol. Rep. 19, 183-194 (1994).

2. Gray, M. W., Burger, G. \& Lang, B. F. Mitochondrial evolution. Science 283, 1476-1481 (1999).

3. Amunts, A., Brown, A., Toots, J., Scheres, S. H. W. \& Ramakrishnan, V. Ribosome. The structure of the human mitochondrial ribosome. Science 348, 95-98 (2015).

4. Greber, B. J. et al. Ribosome. The complete structure of the $55 \mathrm{~S}$ mammalian mitochondrial ribosome. Science 348, 303-308 (2015).

5. Kaushal, P. S. et al. Cryo-EM structure of the small subunit of the mammalian mitochondrial ribosome. Proc. Natl Acad. Sci. USA 111, 7284-7289 (2014).

6. Koripella, R. K. et al. Structures of the human mitochondrial ribosome bound to EF-G1 reveal distinct features of mitochondrial translation elongation. Nat. Commun. 11, 3830 (2020).

7. Koripella, R. K., Sharma, M. R., Risteff, P., Keshavan, P. \& Agrawal, R. K. Structural insights into unique features of the human mitochondrial ribosome recycling. Proc. Natl Acad. Sci. USA 116, 8283-8288 (2019).

8. Sharma, M. R. et al. Structure of the mammalian mitochondrial ribosome reveals an expanded functional role for its component proteins. Cell 115, 97-108 (2003).

9. Christian, B. E. \& Spremulli, L. L. Mechanism of protein biosynthesis in mammalian mitochondria. Biochim Biophys. Acta 1819, 1035-1054 (2012)

10. Sharma, M. R., Kaushal, P. S., Gupta, M., Banavali, N. K. \& Agrawal, R. K. in Translation in Mitochondria and Other Organelles (ed.Duchene, A.-M.) 1-28 (Springer, 2013).

11. Kisselev, L., Ehrenberg, M. \& Frolova, L. Termination of translation: interplay of mRNA, rRNAs and release factors? EMBO J. 22, 175-182 (2003).

12. Poole, E. \& Tate, W. Release factors and their role as decoding proteins: specificity and fidelity for termination of protein synthesis. Biochim. Biophys. Acta 1493, 1-11 (2000).

13. Freistroffer, D. V., Pavlov, M. Y., MacDougall, J., Buckingham, R. H. \& Ehrenberg, M. Release factor RF3 in E.coli accelerates the dissociation of release factors RF1 and RF2 from the ribosome in a GTP-dependent manner. EMBO J. 16, 4126-4133 (1997).

14. Gao, N., Zavialov, A. V., Ehrenberg, M. \& Frank, J. Specific interaction between EF-G and RRF and its implication for GTP-dependent ribosome splitting into subunits. J. Mol. Biol. 374, 1345-1358 (2007).

15. Kaji, A. et al. The fourth step of protein synthesis: disassembly of the posttermination complex is catalyzed by elongation factor $\mathrm{G}$ and ribosome recycling factor, a near-perfect mimic of tRNA. Cold Spring Harb. Symp. Quant. Biol. 66, 515-529 (2001).

16. Karimi, R., Pavlov, M. Y., Buckingham, R. H. \& Ehrenberg, M. Novel roles for classical factors at the interface between translation termination and initiation. Mol. Cell 3, 601-609 (1999).

17. Rao, A. R. \& Varshney, U. Specific interaction between the ribosome recycling factor and the elongation factor $\mathrm{G}$ from Mycobacterium tuberculosis mediates peptidyl-tRNA release and ribosome recycling in Escherichia coli. EMBO J. 20, 2977-2986 (2001).

18. Zavialov, A. V., Hauryliuk, V. V. \& Ehrenberg, M. Splitting of the posttermination ribosome into subunits by the concerted action of RRF and EF-G. Mol. Cell 18, 675-686 (2005).

19. Barat, C. et al. Progression of the ribosome recycling factor through the ribosome dissociates the two ribosomal subunits. Mol. Cell 27, 250-261 (2007).

20. Gao, N. et al. Mechanism for the disassembly of the posttermination complex inferred from cryo-EM studies. Mol. Cell 18, 663-674 (2005).
21. Frank, J. \& Agrawal, R. K. A ratchet-like inter-subunit reorganization of the ribosome during translocation. Nature 406, 318-322 (2000).

22. Borg, A., Pavlov, M. \& Ehrenberg, M. Complete kinetic mechanism for recycling of the bacterial ribosome. RNA 22, 10-21 (2016).

23. Hirokawa, G. et al. The role of ribosome recycling factor in dissociation of $70 \mathrm{~S}$ ribosomes into subunits. RNA 11, 1317-1328 (2005).

24. Peske, F., Rodnina, M. V. \& Wintermeyer, W. Sequence of steps in ribosome recycling as defined by kinetic analysis. Mol. Cell 18, 403-412 (2005).

25. Savelsbergh, A., Rodnina, M. V. \& Wintermeyer, W. Distinct functions of elongation factor $\mathrm{G}$ in ribosome recycling and translocation. RNA 15, 772-780 (2009).

26. Hammarsund, M. et al. Identification and characterization of two novel human mitochondrial elongation factor genes, hEFG2 and hEFG1, phylogenetically conserved through evolution. Hum. Genet. 109, 542-550 (2001).

27. Tsuboi, M. et al. EF-G2mt is an exclusive recycling factor in mammalian mitochondrial protein synthesis. Mol. Cell 35, 502-510 (2009).

28. Bhargava, K., Templeton, P. \& Spremulli, L. L. Expression and characterization of isoform 1 of human mitochondrial elongation factor G. Protein Expr. Purif. 37, 368-376 (2004).

29. Zhang, Y. \& Spremulli, L. L. Identification and cloning of human mitochondrial translational release factor 1 and the ribosome recycling factor. Biochim. Biophys. Acta 1443, 245-250 (1998).

30. Aibara, S., Singh, V., Modelska, A. \& Amunts, A. Structural basis of mitochondrial translation. Elife 9, e58362 (2020).

31. Agrawal, R. K. et al. Visualization of ribosome-recycling factor on the Escherichia coli $70 S$ ribosome: functional implications. Proc. Natl Acad. Sci. USA 101, 8900-8905 (2004)

32. Dunkle, J. A. et al. Structures of the bacterial ribosome in classical and hybrid states of tRNA binding. Science 332, 981-984 (2011).

33. Weixlbaumer, A. et al. Crystal structure of the ribosome recycling factor bound to the ribosome. Nat. Struct. Mol. Biol. 14, 733-737 (2007).

34. Yokoyama, T. et al. Structural insights into initial and intermediate steps of the ribosome-recycling process. EMBO J. 31, 1836-1846 (2012).

35. Zhou, D., Tanzawa, T., Lin, J. \& Gagnon, M. G. Structural basis for ribosome recycling by RRF and tRNA. Nat. Struct. Mol. Biol. 27, 25-32 (2020).

36. Fu, Z. et al. Key intermediates in ribosome recycling visualized by timeresolved cryoelectron microscopy. Structure 24, 2092-2101 (2016).

37. Selmer, M., Al-Karadaghi, S., Hirokawa, G., Kaji, A. \& Liljas, A. Crystal structure of Thermotoga maritima ribosome recycling factor: a tRNA mimic. Science 286, 2349-2352 (1999).

38. Yoshida, T. et al. Solution structure of the ribosome recycling factor from Aquifex aeolicus. Biochemistry 40, 2387-2396 (2001).

39. Connell, S. R. et al. Structural basis for interaction of the ribosome with the switch regions of GTP-bound elongation factors. Mol. Cell 25, 751-764 (2007).

40. Margus, T., Remm, M. \& Tenson, T. Phylogenetic distribution of translational GTPases in bacteria. BMC Genomics 8, 15 (2007).

41. Seshadri, A., Samhita, L., Gaur, R., Malshetty, V. \& Varshney, U. Analysis of the fusA2 locus encoding EFG2 in Mycobacterium smegmatis. Tuberculosis 89, 453-464 (2009).

42. Suematsu, T. et al. A bacterial elongation factor $\mathrm{G}$ homologue exclusively functions in ribosome recycling in the spirochaete Borrelia burgdorferi. Mol. Microbiol. 75, 1445-1454 (2010).

43. Koripella, R. K. et al. Structure of human mitochondrial translation initiation factor 3 bound to the small ribosomal subunit. iScience 12, 76-86 (2019).

44. Kummer, E. et al. Unique features of mammalian mitochondrial translation initiation revealed by cryo-EM. Nature 560, 263-267 (2018).

45. Yassin, A. S. et al. Insertion domain within mammalian mitochondrial translation initiation factor 2 serves the role of eubacterial initiation factor 1 Proc. Natl Acad. Sci. USA 108, 3918-3923 (2011)

46. Kummer, E. \& Ban, N. Structural insights into mammalian mitochondrial translation elongation catalyzed by mtEFG1. EMBO J. 39, e104820 (2020).

47. Gao, Y. G. et al. The structure of the ribosome with elongation factor $\mathrm{G}$ trapped in the posttranslocational state. Science 326, 694-699 (2009).

48. Hirokawa, G. et al. Post-termination complex disassembly by ribosome recycling factor, a functional tRNA mimic. EMBO J. 21, 2272-2281 (2002).

49. Budkevich, T. V. et al. Regulation of the mammalian elongation cycle by subunit rolling: a eukaryotic-specific ribosome rearrangement. Cell 158, 121-131 (2014).

50. Ratje, A. H. et al. Head swivel on the ribosome facilitates translocation by means of intra-subunit tRNA hybrid sites. Nature 468, 713-716 (2010).

51. Schuwirth, B. S. et al. Structures of the bacterial ribosome at 3.5 A resolution. Science 310, 827-834 (2005)

52. Boerema, A. P. et al. Structure of the chloroplast ribosome with chl-RRF and hibernation-promoting factor. Nat. Plants 4, 212-217 (2018).

53. Sharma, M. R. et al. Cryo-EM study of the spinach chloroplast ribosome reveals the structural and functional roles of plastid-specific ribosomal proteins. Proc. Natl Acad. Sci. USA 104, 19315-19320 (2007). 
54. Rorbach, J. et al. The human mitochondrial ribosome recycling factor is essential for cell viability. Nucleic Acids Res. 36, 5787-5799 (2008).

55. AEvarsson, A. et al. Three-dimensional structure of the ribosomal translocase: elongation factor $\mathrm{G}$ from Thermus thermophilus. EMBO J. 13, 3669-3677 (1994).

56. Czworkowski, J., Wang, J., Steitz, T. A. \& Moore, P. B. The crystal structure of elongation factor $\mathrm{G}$ complexed with GDP, at 2.7 A resolution. EMBO J. 13, 3661-3668 (1994).

57. Mears, J. A. et al. A structural model for the large subunit of the mammalian mitochondrial ribosome. J. Mol. Biol. 358, 193-212 (2006).

58. Desai, N. et al. Elongational stalling activates mitoribosome-associated quality control. Science 370, 1105-1110 (2020).

59. Diaconu, M. et al. Structural basis for the function of the ribosomal L7/12 stalk in factor binding and GTPase activation. Cell 121, 991-1004 (2005).

60. Helgstrand, M. et al. The ribosomal stalk binds to translation factors IF2, EF$\mathrm{Tu}, \mathrm{EF}-\mathrm{G}$ and RF3 via a conserved region of the L12 C-terminal domain. J. Mol. Biol. 365, 468-479 (2007).

61. Datta, P. P., Sharma, M. R., Qi, L., Frank, J. \& Agrawal, R. K. Interaction of the $\mathrm{G}$ ' domain of elongation factor $\mathrm{G}$ and the $\mathrm{C}$-terminal domain of ribosomal protein L7/L12 during translocation as revealed by cryo-EM. Mol. Cell 20, 723-731 (2005).

62. Imai, H., Uchiumi, T. \& Kodera, N. Direct visualization of translational GTPase factor pool formed around the archaeal ribosomal P-stalk by highspeed AFM. Proc. Natl Acad. Sci. USA 117, 32386-32394 (2020).

63. Chen, Y., Koripella, R. K., Sanyal, S. \& Selmer, M. Staphylococcus aureus elongation factor G-structure and analysis of a target for fusidic acid. FEBS J. 277, 3789-3803 (2010).

64. Pulk, A. \& Cate, J. H. Control of ribosomal subunit rotation by elongation factor G. Science 340, 1235970 (2013).

65. Fujiwara, T., Ito, K., Nakayashiki, T. \& Nakamura, Y. Amber mutations in ribosome recycling factors of Escherichia coli and Thermus thermophilus: evidence for C-terminal modulator element. FEBS Lett. 447, 297-302 (1999).

66. Eberly, S. L., Locklear, V. \& Spremulli, L. L. Bovine mitochondrial ribosomes. Elongation factor specificity. J. Biol. Chem. 260, 8721-8725 (1985).

67. Zhou, J., Lancaster, L., Donohue, J. P. \& Noller, H. F. How the ribosome hands the A-site tRNA to the P site during EF-G-catalyzed translocation. Science 345, 1188-1191 (2014).

68. Kolesnikov, A. V. \& Gudkov, A. T. Mutational analysis of the functional role of the loop region in the elongation factor $\mathrm{G}$ fourth domain in the ribosomal translocation. Mol. Biol. 37, 719-725 (2003).

69. Zhang, D. et al. New insights into the enzymatic role of EF-G in ribosome recycling. Nucleic Acids Res. 43, 10525-10533 (2015).

70. Chen, Y., Feng, S., Kumar, V., Ero, R. \& Gao, Y. G. Structure of EF-G-ribosome complex in a pretranslocation state. Nat. Struct. Mol. Biol. 20, 1077-1084 (2013).

71. Tourigny, D. S., Fernandez, I. S., Kelley, A. C. \& Ramakrishnan, V. Elongation factor $\mathrm{G}$ bound to the ribosome in an intermediate state of translocation. Science 340, 1235490 (2013).

72. Zhou, J., Lancaster, L., Donohue, J. P. \& Noller, H. F. Crystal structures of EFG-ribosome complexes trapped in intermediate states of translocation. Science 340, 1236086 (2013).

73. Chung, H. K. \& Spremulli, L. L. Purification and characterization of elongation factor $\mathrm{G}$ from bovine liver mitochondria. J. Biol. Chem. 265 , 21000-21004 (1990).

74. Lee, M. et al. Reconstitution of mammalian mitochondrial translation system capable of correct initiation and long polypeptide synthesis from leaderless mRNA. Nucleic Acids Res. 49, 371-382 (2020).

75. Mondal, S., Hsiao, K. \& Goueli, S. A. A homogenous bioluminescent system for measuring GTPase, GTPase activating protein, and guanine nucleotide exchange factor activities. Assay Drug Dev. Technol. 13, 444-455 (2015).

76. Punjani, A., Rubinstein, J. L., Fleet, D. J. \& Brubaker, M. A. cryoSPARC: algorithms for rapid unsupervised cryo-EM structure determination. Nat. Methods 14, 290-296 (2017).

77. Rohou, A. \& Grigorieff, N. CTFFIND4: fast and accurate defocus estimation from electron micrographs. J. Struct. Biol. 192, 216-221 (2015).

78. Pettersen, E. F. et al. UCSF Chimera-a visualization system for exploratory research and analysis. J. Comput. Chem. 25, 1605-1612 (2004).
79. Adams, P. D. et al. PHENIX: a comprehensive Python-based system for macromolecular structure solution. Acta Crystallrogr. D Biol. Crystallogr. 66, 213-221 (2010)

80. Roy, A., Kucukural, A. \& Zhang, Y. I-TASSER: a unified platform for automated protein structure and function prediction. Nat. Protoc. 5, 725-738 (2010).

81. Emsley, P., Lohkamp, B., Scott, W. G. \& Cowtan, K. Features and development of Coot. Acta Crystallogr. D Biol. Crystallogr. 66, 486-501 (2010).

82. Chen, V. B. et al. MolProbity: all-atom structure validation for macromolecular crystallography. Acta Crystallogr. D. Biol. Crystallogr. 66, 12-21 (2010)

83. Goddard, T. D. et al. UCSF ChimeraX: meeting modern challenges in visualization and analysis. Protein Sci. 27, 14-25 (2018).

\section{Acknowledgements}

We thank Linda Spremulli (UNC), and Robert Lightowlers and Zofia ChrzanowskaLightowlers (New Castle, UK) for sharing their RRFmt and EF-G2mt clones. We thank Manjuli Sharma for helpful suggestions and comments during the course of this study and on the manuscript. We acknowledge the use of the Wadsworth Center's Media and Culture core facility, for help in producing large volumes of HEK293S GnTI cells, and the Wadsworth Center's and New York Structural Biology Center's (NYSBC's) EM facilities NYSBC EM facilities are supported by grants from the Simons Foundation (349247), NYSTAR, the NIH (GM103310) and the Agouron Institute (F00316). This work was supported by the NIH grant R01 GM61576 (to R.K.A.).

\section{Author contributions}

R.K.A. conceived this study. P.K. purified human mitoribosomes, human RRFmt and EFG2mt, and R.K.K. prepared the human 55S PoTC.RRFmt•EF-G2mt complex. R.K.K. and N.K.B. collected cryo-EM data, and R.K.K. and R.K.A. performed image processing. R.K. K. performed molecular modeling, using assistance from A.D., E.K.A., and N.K.B. A.D., E.K.A., and P.K. performed the biochemical assays. R.K.K., A.D., and R.K.A. analyzed the data and wrote the manuscript. All authors read and approved the manuscript. Authors declare no competing financial interest in this work.

\section{Competing interests}

The authors declare no competing interests.

\section{Additional information}

Supplementary information The online version contains supplementary material available at https://doi.org/10.1038/s41467-021-23726-4.

Correspondence and requests for materials should be addressed to R.K.A.

Peer review information Nature Communications thanks Yaser Hashem, Suparna Sanyal and other, anonymous, reviewers for their contributions to the peer review of this work.

Reprints and permission information is available at http://www.nature.com/reprints

Publisher's note Springer Nature remains neutral with regard to jurisdictional claims in published maps and institutional affiliations.

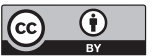

Open Access This article is licensed under a Creative Commons Attribution 4.0 International License, which permits use, sharing, adaptation, distribution and reproduction in any medium or format, as long as you give appropriate credit to the original author(s) and the source, provide a link to the Creative Commons license, and indicate if changes were made. The images or other third party material in this article are included in the article's Creative Commons license, unles indicated otherwise in a credit line to the material. If material is not included in the article's Creative Commons license and your intended use is not permitted by statutory regulation or exceeds the permitted use, you will need to obtain permission directly from the copyright holder. To view a copy of this license, visit http://creativecommons.org/ licenses/by/4.0/

(C) The Author(s) 2021 